\title{
Eddy-Induced Particle Dispersion in the Near-Surface North Atlantic
}

\author{
IRINA I. RYPINA \\ Physical Oceanography Department, Woods Hole Oceanographic Institution, Woods Hole, Massachusetts \\ IGOR KAMENKOVICH \\ Division of Meteorology and Physical Oceanography, Rosenstiel School of Marine and Atmospheric Science, \\ University of Miami, Miami, Florida \\ PAVEL BERLOFF \\ Grantham Institute for Climate Change and Department of Mathematics, Imperial College London, South \\ Kensington Campus, London, United Kingdom \\ LAWRENCE J. PRATT \\ Physical Oceanography Department, Woods Hole Oceanographic Institution, Woods Hole, Massachusetts
}

(Manuscript received 19 October 2011, in final form 20 July 2012)

\begin{abstract}
This study investigates the anisotropic properties of the eddy-induced material transport in the near-surface North Atlantic from two independent datasets, one simulated from the sea surface height altimetry and one derived from real-ocean surface drifters, and systematically examines the interactions between the mean- and eddy-induced material transport in the region. The Lagrangian particle dispersion, which is widely used to characterize the eddy-induced tracer fluxes, is quantified by constructing the "spreading ellipses." The analysis consistently demonstrates that this dispersion is spatially inhomogeneous and strongly anisotropic. The spreading is larger and more anisotropic in the subtropical than in the subpolar gyre, and the largest ellipses occur in the Gulf Stream vicinity. Even at times longer than half a year, the spreading exhibits significant nondiffusive behavior in some parts of the domain. The eddies in this study are defined as deviations from the long-term time-mean. The contributions from the climatological annual cycle, interannual, and subannual (shorter than one year) variability are investigated, and the latter is shown to have the strongest effect on the anisotropy of particle spreading. The influence of the mean advection on the eddy-induced particle spreading is investigated using the "eddy-following-full-trajectories" technique and is found to be significant. The role of the Ekman advection is, however, secondary. The pronounced anisotropy of particle dispersion is expected to have important implications for distributing oceanic tracers, and for parameterizing eddy-induced tracer transfer in non-eddy-resolving models.
\end{abstract}

\section{Introduction}

\section{a. Background}

The mesoscale oceanic eddies ("eddies" hereafter) are important for maintaining the oceanic large-scale stratification, general circulation, and distribution of bio and geo-chemical tracers. One of the most important

Corresponding author address: Irina I. Rypina, Physical Oceanography Department, Woods Hole Oceanographic Institution, Woods Hole, MA 02543.

E-mail: irypina@whoi.edu features of the eddies is their ability to transport material properties-this process is commonly modeled as eddy diffusion. The eddy diffusivity parameter quantifies the efficiency of eddies in downgradient mixing of the large-scale properties. The diffusivity can be estimated from Lagrangian float statistics (e.g., Owens 1984; Davis 1991; see a review in LaCasce 2008). Horizontal or isopycnal diffusion has been commonly used to parameterize lateral transport and mixing by the eddies in noneddy-resolving numerical models, which are standard oceanic components of present-day comprehensive climate models. In these models, the large scale advection 
is simulated explicitly, whereas the eddy-induced tracer transport has to be parameterized. The corresponding eddy diffusivities are poorly known and often taken to be isotropic and spatially homogeneous.

However, the existing evidence based on observations and numerical eddy-resolving simulations shows significant complexity in the structure and spatial distribution of the eddy-induced transport properties. The eddy diffusivities estimated directly from the floats (LaCasce and Bower 2000; Lumpkin et al. 2002; McClean et al. 2002) and indirectly from the satellite data (Marshall et al. 2006) have strong geographical inhomogeneities. Eddy-resolving simulations also demonstrate highly nonuniform spatial distribution of the eddy-induced transport (Gille and Davis 1999; Nakamura and Chao 2000; Roberts and Marshall 2000). This complexity of the eddy-induced transport can be very important for simulating distributions of oceanic tracers and properties, as well as for airsea exchanges (e.g., Booth and Kamenkovich 2008). The diffusivity coefficients are also length scale-dependent according to Okubo (1971).

The lateral eddy-induced spreading is also anisotropic, and examples include differences between the alongand cross-stream directions in the Antarctic Circumpolar Current (Marshall et al. 2006; Sallee et al. 2008; Griesel et al. 2010; Ferrari and Nikurashin 2010; Naveira Garabato et al. 2011), and between the zonal and meridional directions in the North Atlantic (McClean et al. 2002) and the tropical Pacific (Bauer et al. 2002). In the North Atlantic, Kamenkovich et al. (2009a) found that zonal spreading rates are several times larger than the meridional ones. This study shows that this anisotropy is more significant in the subtropical than in the subpolar gyre and that it is caused primarily by the transient eddies rather than by the time-mean zonal jets (Maximenko et al. 2005; Berloff et al. 2009, 2011). Such anisotropic mixing can have potentially important implications for the tracer distribution in the ocean (Armi and Haidvogel 1982). Finally, in many locations the spreading rate can be faster or slower than diffusion (Berloff et al. 2002; Veneziani et al. 2005).

Physical mechanisms of the anisotropic spreading are unclear. LaCasce and Speer (1999) found that spreading of particles in idealized barotropic flows is mostly along the contours of $\mathrm{f} / \mathrm{H}$, with water depth $\mathrm{H}$ and Coriolis parameter $\mathrm{f}$. Considerable $\mathrm{f} / \mathrm{H}$ control on the observed floats was further reported, and the difference between the spreadings along and across $\mathrm{f} / \mathrm{H}$ contours was found greater than difference between zonal and meridional spreadings (LaCasce 2000). On the other hand, O'Dwyer et al. (2000) demonstrated enhanced dispersion of floats in the direction parallel to the contours of the time-mean potential vorticity (PV) (these contours are, however, nearly zonal in the studied location). Another reason for the anisotropy is that the eddy shapes can be highly anisotropic (e.g., Huang et al. 2007; Scott et al. 2008; Berloff et al. 2009), and zonally elongated eddies result in preferentially zonal eddy-induced transport (Kamenkovich et al. 2009a). Effects of the mean large-scale currents can also play an important role in anisotropic eddy transport (Smith 2005; Ferrari and Nikurashin 2010; Rypina et al. 2007a, 2011).

To summarize, quantifying the eddy-induced particle dispersion is critical not only for understanding the kinematic properties of eddies and the importance of eddies in distributing oceanic tracers, but also for accurate modeling of these eddy effects in low-resolution climate models. Recent studies, which employ a diverse set of data, techniques, and numerical models and focus on selected geographical regions, suggest that this eddy-induced transport is spatially inhomogeneous, anisotropic, and even nondiffusive but overall its properties throughout the World Ocean and its underlying physics are poorly understood. With this as our motivation, we have quantified the nearsurface eddy diffusivities and its anisotropic properties in the whole North Atlantic using two independent observational datasets, and systematically examined the interactions between the mean advection and eddy-induced material transport in the region, as well as the contributions from the annual cycle, interannual, and subannual variability.

\section{b. Datasets}

In this study we describe the systematic analysis of Lagrangian trajectories from two independent datasets: trajectories simulated using the near-surface geostrophic currents estimated from satellite altimetry data and observed trajectories of real surface drifters from the Global Drifter Program (GDP) (http://www.aoml.noaa.gov/ phod/dac/index.php). More specifically, the eddy fields used in our simulations are based on altimetric sea surface height anomalies [i.e., Maps of Sea Level Anomalies (MSLA) fields available from http://www.aviso.oceanobs. com/es/data/products/sea-surface-height-products/global/ msla/index.html] that were converted to velocities using geostrophic relation, $\psi=g h(x, y, z) / f$, where $\psi$ is the velocity streamfunction, $g$ is the gravitational acceleration, $f$ is the Coriolis parameter, and $h$ is the sea surface height anomaly. The mean velocities that we used are from the combined Mean Dynamic Topography (MDT) estimate (http://www.aviso.oceanobs.com/es/data/products/auxiliaryproducts/mdt/index.html). These velocities have resolved flow features on spatial scales of about 100-200 km and larger (Chelton et al. 2011). This corresponds to scales of several local first deformation radii: from about $4 R d_{1}$ in the southern part of the subtropical gyre to more than 
TABLE 1. Table describing different numerical simulations and methods used to produce figures.

\begin{tabular}{lcc}
\hline \hline $\begin{array}{c}\text { Run name } \\
\text { (abbreviation) }\end{array}$ & Description & Method \\
\hline Eddy-only (EO) & $\begin{array}{c}\text { Spreading due to eddies without } \\
\text { mean advection } \\
\text { Spreading due to eddies along } \\
\text { mean trajectories }\end{array}$ & $\begin{array}{c}\text { Analyze spreading of particles in simulations } \\
\text { without the time-mean flow } \\
\text { Integrate eddy-only diffusivities along } \\
\text { mean trajectories }\end{array}$ \\
$\begin{array}{c}\text { Eddy-following-full-trajectory } \\
\text { (EFFT) }\end{array}$ & $\begin{array}{c}\text { Spreading due to eddies along } \\
\text { full trajectories }\end{array}$ & $\begin{array}{c}\text { Calculate full trajectories, at each step } \\
\text { subtract displacement due to the mean, } \\
\text { cumulatively add the residual displacements } \\
\text { together to get pseudotracks, and analyze } \\
\text { spreading of pseudotracks }\end{array}$ \\
\hline
\end{tabular}

$8 R d_{1}$ in the northern part of the subpolar gyre. The resolved temporal scales are from one week and longer. As discussed in a companion paper (Kamenkovich et al. 2012, manuscript submitted to J. Phys. Oceanogr.), there are reasons to expect that eddy-induced transport is dominated by eddies with spatial scales longer than $8 R d_{1}$ and time scales longer than week-this is in accord with our study (see also Keating et al. 2011). We also investigate the effects of the Ekman velocities on particle dispersion by adding to the altimetric fields the Ekman velocities obtained from National Aeronautics and Space Administration (NASA)'s Quick Scatterometer (QuikSCAT) wind stresses.

The paper is organized as follows. In section 2 we introduce single-particle dispersion, which is a useful conventional measure of eddy-induced transport. In section 3 we analyze Lagrangian particles in the simulations without the time-mean flow component ("eddy-only" case), and investigate the effects of the annual cycle and interannual variablity on particle dispersion. The effect of the timemean currents on the eddy-induced transport is studied in section 4. A description of different numerical simulations along with their names, methods and resulting figures is presented in Table 1 . In section 5 we compare the analyses of simulated Lagrangian trajectories and real drifter trajectories. The influence of the Ekman velocities on the particles is studied at the end of section 5. The discussion and concluding remarks follow in section 6.

\section{Methodology}

We define the zonal and meridional single-particle dispersions, for an ensemble of $\mathrm{N}$ particles released at the same location at times separated by constant time interval, as

$D_{x}=\frac{1}{N} \sum_{n=1}^{N}\left[x_{n}(t)-X(t)\right]^{2}, \quad D_{y}=\frac{1}{N} \sum_{n=1}^{N}\left[y_{n}(t)-Y(t)\right]^{2}$, where $x_{n}(t), y_{n}(t)$ are, respectively, the zonal and meridional displacements of particles from their initial positions and $X(t)=(1 / N) \sum_{1}^{N} x_{n}(t), Y(t)=(1 / N) \sum_{1}^{N} y_{n}(t)$ are the zonal and meridional displacements of the "centre of mass" of the ensemble (Kamenkovich et al. 2009a). The zonal and meridional spreading rates for the group of particles are defined as

$$
K_{x}=\frac{1}{2} \frac{\partial D_{x}}{\partial t}, \quad K_{y}=\frac{1}{2} \frac{\partial D_{y}}{\partial t} .
$$

In the diffusive spreading regime, dispersions $D_{x}, D_{y}$ grow linearly with time and the corresponding spreading rates $K_{x}=$ const $_{x}$ and $K_{y}=$ const $_{y}$ are referred to as the eddy diffusivity coefficients. Alternatively, the eddy diffusivities can be obtained without explicitly calculating single-particle dispersions by computing the correlation between the velocity and displacement (Davis 1991; Zhurbas and Oh 2004) or by integrating the velocity autocorrelation function $K_{x y y}=\int_{0}^{t} R_{x y y}(\tau) d \tau$, where $R_{x y}(\tau)=\left\langle u_{x y}(t) u_{x y y}(t+\tau)\right\rangle$ (Davis 1991; Sallee et al. 2008; Griesel et al. 2010). Although some of these methods may be more convenient in a particular situation, all of them are expected to lead to similar results. In particular, we rely in this study on the direct, single-particle dispersion-based method of estimating eddy diffusivities, but the velocity autocorrelation method was shown to result in nearly the same diffusivity estimates. All these Lagrangian trajectory-based techniques result in nonlocal estimates of diffusivity. We will come back to this issue in the following sections.

Another way of estimating the eddy-induced diffusivity is by using the method of Nakamura (1996), which has been pioneered for applications to the ocean by Marshall et al. (2006). The method characterizes eddy effects by looking at the elongation of tracer concentration contours, as the tracer is being advected by the flow field. Effective diffusivity yields an estimate of the cross-streamline eddy diffusivity, averaged along the streamlines. Since the effective diffusivity is 
a scalar, it is not well suited for applications where diffusivity is anisotropic, that is, varies with directions, and thus has to be represented as a tensor. This technique is also fundamentally nonlocal, as it involves averaging in the along-streamline direction. The effective diffusivity technique has been most commonly applied to the Southern Ocean. There, the along-stream spreading of a tracer can be expected to be dominated by the mean advection rather than the eddy-induced diffusion. Thus, the use of the effective diffusivity, which characterizes the average cross-stream diffusivity, can be appropriate there. In the North Atlantic, however, both the along- and cross-streamline components of diffusivity are important in large parts of the region so the effective diffusivity is not well-suited for this application. In particular, a simple scaling analysis reveals that the advection term in the tracer balance scales like $U \Delta C / L$ and diffusion termlike $K \Delta C /\left(L^{2}\right)$, where $L$ and $U$ are the characteristic length and velocity scales, and $\Delta C$ is the scale for tracer anomaly. Thus, the advection dominates over diffusion in the tracer distribution when $U \gg K / L$ or, equivalently, when the Peclet number $\mathrm{Pe}=U L / K \gg 1$, which, assuming that the typical values of $L$ and $K$ in the North Atlantic are on the order of $200 \mathrm{~km}$ and $10^{3}$ to $10^{4} \mathrm{~m}^{2}$ $\mathrm{s}^{-1}$, is questionable over large parts of the domain.

Spreading ellipses, angle of maximum spreading, and anisotropy coefficient

In general time-dependent flows, where particle dispersion properties are isotropic and spatially homogeneous, a group of $N$ Lagrangian particles disperses uniformly in all directions and, thus, spreads in a circle. However, real flows often exhibit anisotropic transport properties (see section 1a), where particles spread preferentially in one direction, forming an approximate ellipse. The orientation and shape of such a spreading ellipse can be used to quantify the anisotropy of particle spreading. We denote the angle between the zonal direction and the direction of the fastest spreading by $\phi$, so the dispersion of particles in the latter direction, $D_{\tau}$, and in the perpendicular direction, $D_{n}$, can be expressed as

$$
\begin{aligned}
& D_{\tau}=D_{x} \cos ^{2}(\phi)+D_{y} \sin ^{2}(\phi)+D_{x y} \sin (2 \phi), \text { and } \\
& D_{n}=D_{x} \sin ^{2}(\phi)+D_{y} \cos ^{2}(\phi)-D_{x y} \sin (2 \phi),
\end{aligned}
$$

where

$$
D_{x y}=\frac{1}{N} \sum_{1}^{N}\left[x_{n}(t)-X(t)\right]\left[y_{n}(t)-Y(t)\right] .
$$

If $D_{x}, D_{y}$, and $D_{x y}$ are known, then $\phi$ can be estimated by maximizing (3) with respect to $\phi$, that is, by choosing $\phi$ at which $D_{\tau}$ is the largest:

$$
\tan (\phi)=\frac{D_{y}-D_{x}+\sqrt{\left(D_{y}-D_{x}\right)^{2}+4 D_{x y}}}{2 D_{x y}}
$$

The $\tau$ and $n$ components of $K$, as well as the corresponding angle $\phi$ at which $K_{\tau}$ is the largest, are also given by (3), (4), and (6), but where $K$ is substituted for $D$. Alternatively, $K_{\tau}$ and $K_{n}$ can be expressed through velocity correlations:

$$
\begin{aligned}
& K_{\tau}=\int_{0}^{t} d t\left\{R_{x}(t) \cos ^{2}(\phi)+R_{y}(t) \sin ^{2}(\phi)+\left[R_{x y}(t)+R_{x y}(-t)\right] \frac{\sin (2 \phi)}{2}\right\}, \quad \text { and } \\
& K_{n}=\int_{0}^{t} d t\left\{R_{x}(t) \sin ^{2}(\phi)+R_{y}(t) \cos ^{2}(\phi)-\left[R_{x y}(t)+R_{x y}(-t)\right] \frac{\sin (2 \phi)}{2}\right\}
\end{aligned}
$$

In this paper, we will portray eddy-induced transport properties using the "spreading ellipses" (single-particle dispersion ellipses), characterized by the major and minor axes, $a=D_{\tau}$ and $b=D_{n}$, respectively, and by the slope (or inclination angle), $\phi$, corresponding to the direction of the fastest spreading. This technique is similar to that used by O'Dwyer et al. (2000) who reported dispersion ellipses at a few locations in the North Atlantic. The ratio $R_{D}=D_{\tau} / D_{n}$ is referred to as the anisotropy coefficient. The ellipses with $a=K_{\tau}, b=K_{n}$ and slope $\phi$ corresponding to the direction in which $K_{\tau}$ is the largest will be referred to as the "spreading rate ellipses" or "diffusivity ellipses."

\section{3. "Eddy-only" (EO) simulations based on altimetry}

We begin our analysis by looking at the spatial inhomogeneity and anisotropic properties of the eddy field. The eddies are defined here in the most straightforward way, as deviations from the long-term time mean. A plot of the 16-yr average eddy kinetic energy 


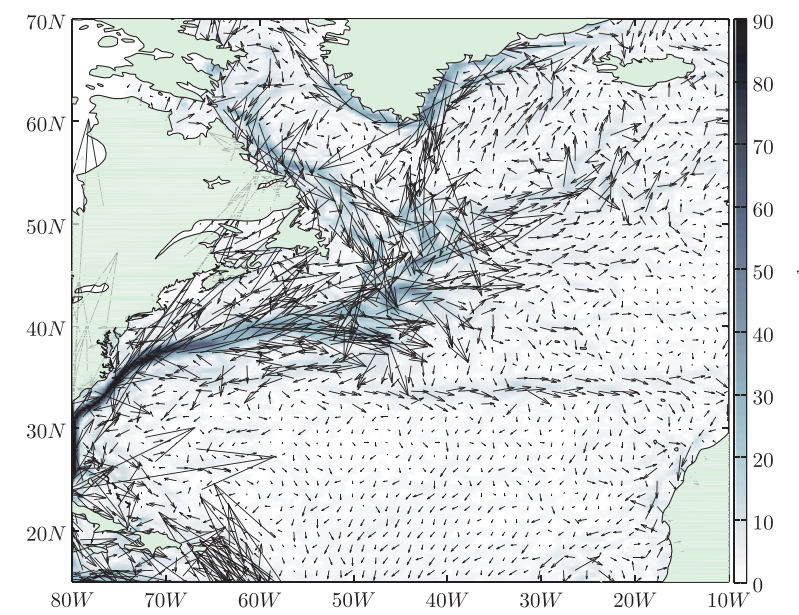

(a)

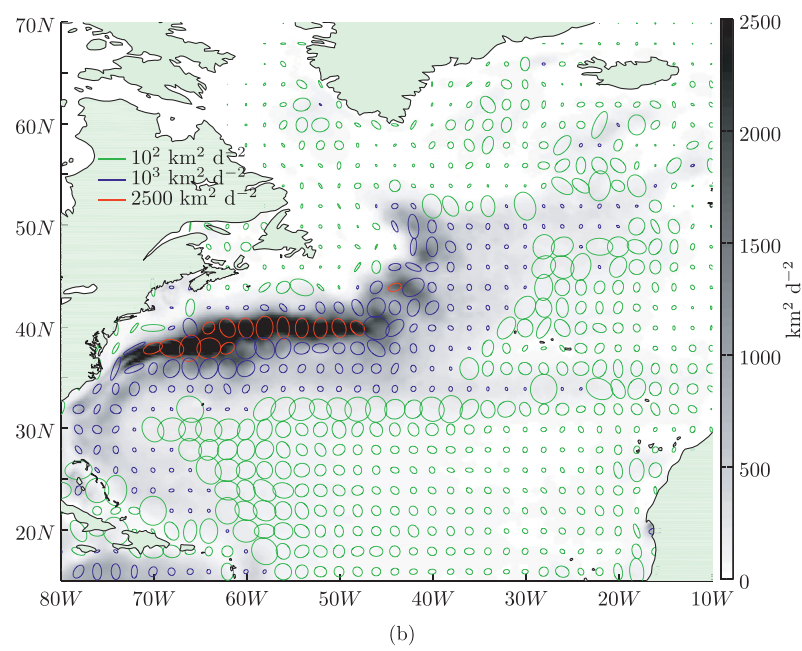

FIG. 1. (a) Mean circulation corresponding to the mean dynamic topography; and (b) time-averaged eddy kinetic energy computed from altimetric sea surface heights (grayscale) and EKE ellipses (color). Scaling for EKE ellipses is indicated by the red, blue and green segments and associated numbers next to them.

(EKE) together with the corresponding velocity covariance ellipses (or EKE ellipses, see Preisendorfer 1988; Morrow et al. 1994; Sallee et al. 2008) are shown in Fig. 1b. The orientation of the EKE ellipses, defined through maximizing $\operatorname{EKE}_{\tau}=\left\langle u^{2}\right\rangle \cos ^{2} \tilde{\phi}+\left\langle v^{2}\right\rangle \sin ^{2} \tilde{\phi}+$ $\langle u v\rangle \sin 2 \tilde{\phi}$ with respect to $\tilde{\phi}$, shows the direction of the maximum velocity covariance and the ratio between the major and minor ellipse axes characterizes the anisotropy of the eddy field. The ellipses are only mildly anisotropic throughout most of the North Atlantic with the domain-averaged anisotropy coefficient $<2$. As we will see below, the eddy-induced material transport is significantly more anisotropic.

As the first step in analyzing the eddy-induced transport, we estimated the dispersion of simulated particles advected by the eddy fields only (without the time mean).

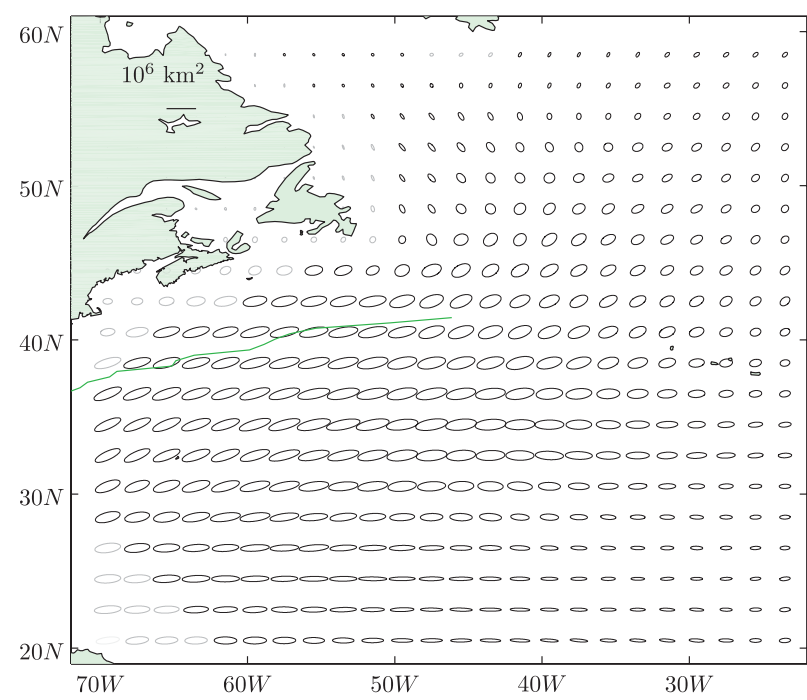

FIG. 2. Spatial distribution of the eddy-induced eddy-only spreading ellipses. Ellipses are from the simulated particles advected by altimetric eddy field. Particles were released once per month from October 1992 until July 2007 and were tracked for 1.5 years; the spreading ellipses were calculated at the end of $1.5-\mathrm{yr}$ simulation. Gray color indicates bins where more than $40 \%$ of the trajectories are affected by the boundary. Green curve shows the mean Gulf Stream core.

For this purpose, the North Atlantic domain was divided into $1^{\circ} \times 1^{\circ}$ bins, and in each bin groups of 100 uniformly distributed particles were released once per month (from October 1992 until July 2007) and tracked for 1.5 years. According to (1), the resulting particle trajectories in each bin were used to calculate time-average $D_{x}, D_{y}$, and $D_{x y}$ and to estimate the angle of maximum spreading, $\phi$. With $\phi, D_{\tau}$, and $D_{n}$, we then plotted spreading ellipses for all bins (Fig. 2).

Figure 2 clearly demonstrates that the eddy-induced particle dispersion is highly anisotropic, and that the dispersion values and the spatial distribution of both $\phi$ and the degree of the anisotropy, $R_{D}$, are all highly nonuniform. The largest ellipses are in the western subtropical gyre and in the Gulf Stream eastward extension region, between approximately $32^{\circ}$ and $42^{\circ} \mathrm{N}$, where the EKE is also the largest (Fig. 1). In particular, the spreading is predominantly zonal in the southern part of the subtropical gyre and nonzonal in the vicinity of the intense currents. The spreading ellipses are tilted by about $+20^{\circ}$ in the Gulf Stream extension region and by about $-60^{\circ}$ in the western part of the subpolar gyre. To alleviate the influence of the boundaries in steering the particle trajectories, we identified the bins with more than $40 \%$ of all trajectories passing within $100 \mathrm{~km}$ from the coast during 1.5 years (ellipses shown by gray). Note that in the northwestern subtropical and western subpolar gyres these ellipses are aligned with the coast, suggesting 
that the anisotropic spreading in these areas may be partially explained by the influence of the boundaries. The degree of anisotropy is generally large $\left(R_{D}=D_{\tau} / D_{n} \gtrsim 3.5\right)$ throughout the subtropical gyre, and it decreases to $\sim 1.8$ in the subpolar gyre. These values are in a good agreement with an eddy-resolving general circulation model of the North Atlantic (Kamenkovich et al. 2009a), where the ratios of zonal to meridional EO dispersions $\left(D_{x} / D_{y}\right)$ at the ocean surface are about 5 and 1.5, respectively, for the subtropical and subpolar gyres. Note also that these spreading ellipses are significantly different from the EKE ellipses reported in Fig. 1b.

The spreading ellipses presented in Fig. 2 are spatially nonlocal in the sense that the spreading of particles from each bin is affected by the eddy field in a much larger than this bin area. In other words, as initially nearby particles spread apart, the spreading ellipses become larger and begin to overlap with each other. To account for this effect, the effective "mean" spreading ellipse for each bin can be obtained by averaging over all ellipses that cover this bin. By computing $D_{\tau, n}$ and $\phi$ for the "mean" ellipses (averaged over all of the overlapping ellipses), we found that this averaging effectively removes some spatial variability from the spreading estimates. Despite this smoothing effect, the "mean" spreading ellipses (not shown) are very similar to the original ones (Fig. 2), therefore, in the rest of the analysis, this averaging is not used.

In our definition of the eddy field that we used to produce Figs. 1 and 2, the eddy field includes the climatological annual cycle, as well as deviations from the climatology on the time scales of shorter (subannual), and longer (interannual) than one year. We now investigate the contribution of the annual cycle and the interannual variability on both the EKE ellipses and particle spreading. For this purpose, we have carried out simulations with eddy velocities from which we removed 1) the annual cycle, and 2) the annual cycle and the interannual variability (to filter out the interannual variability we highpassed the eddy velocities using the Chebyshev window with the cut-off period of 420 days). The results are shown in Fig. 3 for the EKE ellipses and in Fig. 4 for the spreading ellipses. Both figures suggest that the effect of the annual cycle is very small and its removal does not lead to any significant changes in either the EKE ellipses or the spreading ellipses. Removal of the interannual variability causes a more interesting effect: local EKE decreases by about $8 \%-30 \%$ throughout the domain with the exception of one area in the eastern North Atlantic, where much larger EKE decrease, up to $70 \%$, is seen (see Fig. 3b for the details of the spatial distribution of $\left.\mathrm{EKE}_{\text {unfiltered }} / \mathrm{EKE}_{\text {filtered }}\right)$. The changes in the EKE anisotropy coefficient caused by the removal of interannual variability are presented in Fig. 3c, where we show the ratio of the unfiltered to filtered EKE anisotropy coefficients. With the removal of the interannual variability, the anisotropy coefficient stays largely unchanged with the small increase in the southeastern North Atlantic, and the decrease in the northeast and in the vicinity of the western boundary currents. The EO spreading ellipses also change when we remove the interannual variability (Fig. 3), but the pattern here is different from the changes in EKE. The ellipses stay largely unchanged in the general area of the Gulf Stream and its extension, but become smaller and more isotropic in the southern North Atlantic and in the western subpolar region. Interestingly, the area of the largest EKE changes centered around $25^{\circ} \mathrm{W}$ and $45^{\circ} \mathrm{N}$ in Fig. $3 \mathrm{~b}$ is not observed in Fig. 4b. Three conclusions can be drawn from this analysis. First, the influence of the climatological annual cycle is negligible. Second, the structure of the singleparticle spreading ellipses is drastically different, and thus cannot be directly inferred, from the EKE ellipses. And third, even after the removal of the annual cycle and interannual variability, the spreading ellipses are still strongly anisotropic, suggesting that the anisotropy is primarily caused by mesoscale eddies. In the remainder of the paper, we will be analyzing spreading by unfiltered velocities.

How diffusive is the spreading? Before answering this question, let us clarify our subsequent use of terms "subdiffusive/superdiffusive" behavior. Although this terminology is widely used in the literature (see, for example, Berloff et al. 2002 and references therein), the meaning of these terms can be different for flows that are locally (in the Eulerian sense) nondiffusive and flows that are nondiffusive in the Lagrangian sense. Since the singleparticle dispersion $D$ and the corresponding diffusivity $K$ characterize the spread of an ensemble of particles away from their launch position, both $D$ and $K$ are Lagrangian in nature and thus intrinsically nonlocal. Thus, we can only say that the Lagrangian particle spreading is subdiffusive/ superdiffusive, that is, the growth of $D(t)$ in time is slower or faster than linear, but this does not necessarily mean that the process is locally nondiffusive. For instance, if the eddy field is strongly spatially inhomogeneous, the growth of $D(t)$ in time can be significantly different from linear even if the eddy field is purely diffusive locally in the Eulerian sense. We will illustrate this point on a particular example later in this section.

That said, the temporal analysis of the dispersion regimes is presented in Fig. 5. The diffusive regime corresponds to the linear growth of dispersion with time, and this behavior shows up on a log-log plot as a line with the slope of unity. Deviation of the slope from unity then quantifies how nondiffusive the spreading is. For 


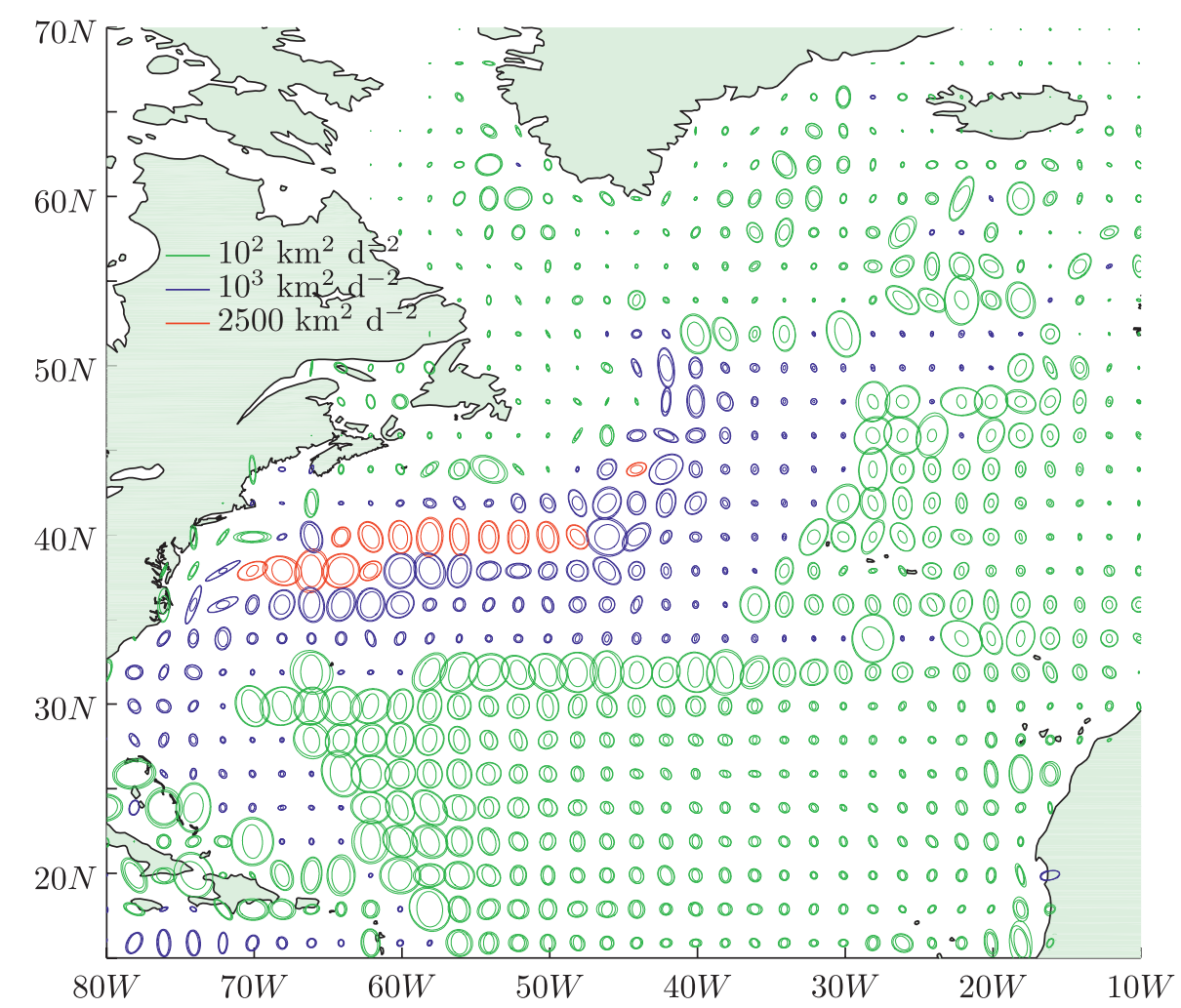

(a)

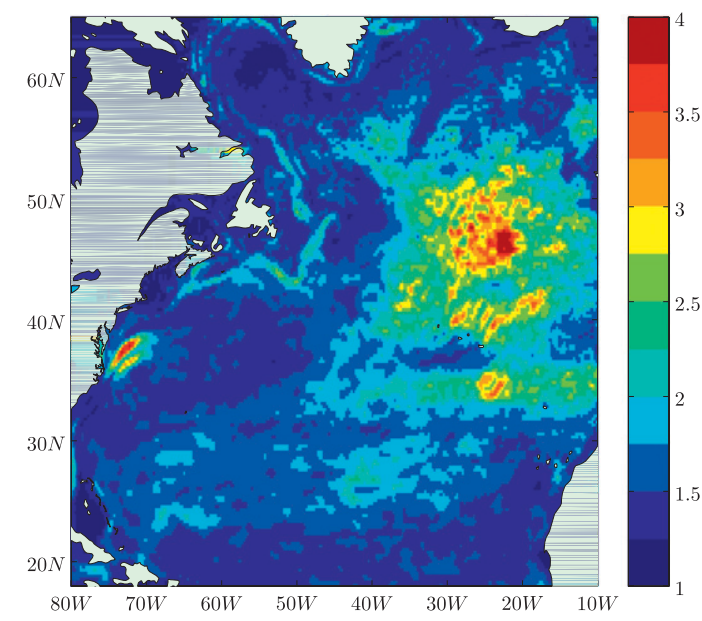

(b)

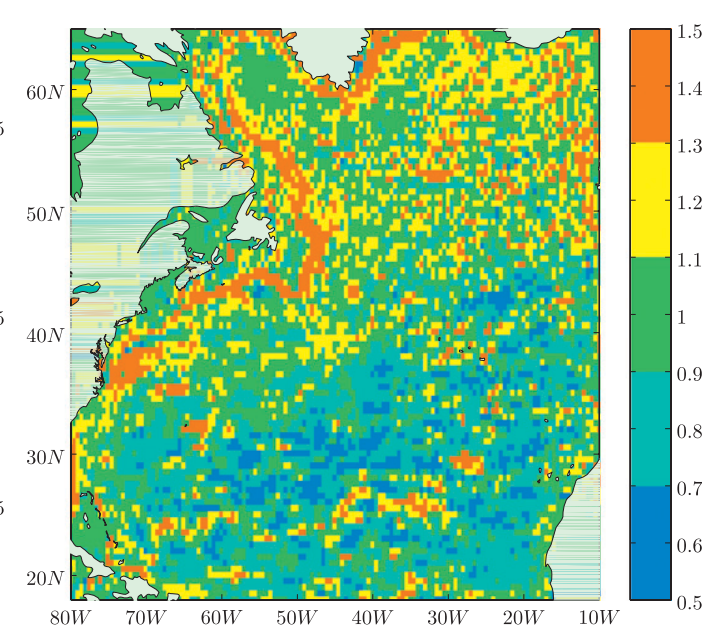

(c)

FIG. 3. (a) EKE ellipses for the unfiltered eddy field (largest ellipses), eddy field with removed annual cycle (intermediate ellipses), and eddy field with removed annual cycle and interannual variability (smallest ellipses). Colors as in Fig. 1b. (b) Ratio of the unfiltered and filtered (without annual cycle and interannual variability) EKE. (c) Ratio of the unfiltered and filtered EKE anisotropy coefficient.

example, values larger and smaller than unity correspond to super and subdiffusive regimes, respectively. Note that the nature of spreading changes with time (Fig. 5), and the characterization of the regime as diffusive, sub- or superdiffusive should be done after sufficient time has passed since the particle deployment. For example, consider an idealized case of an ensemble of particles that were released simultaneously very close to each other. Subsequent spreading of the particles will go through several stages. 


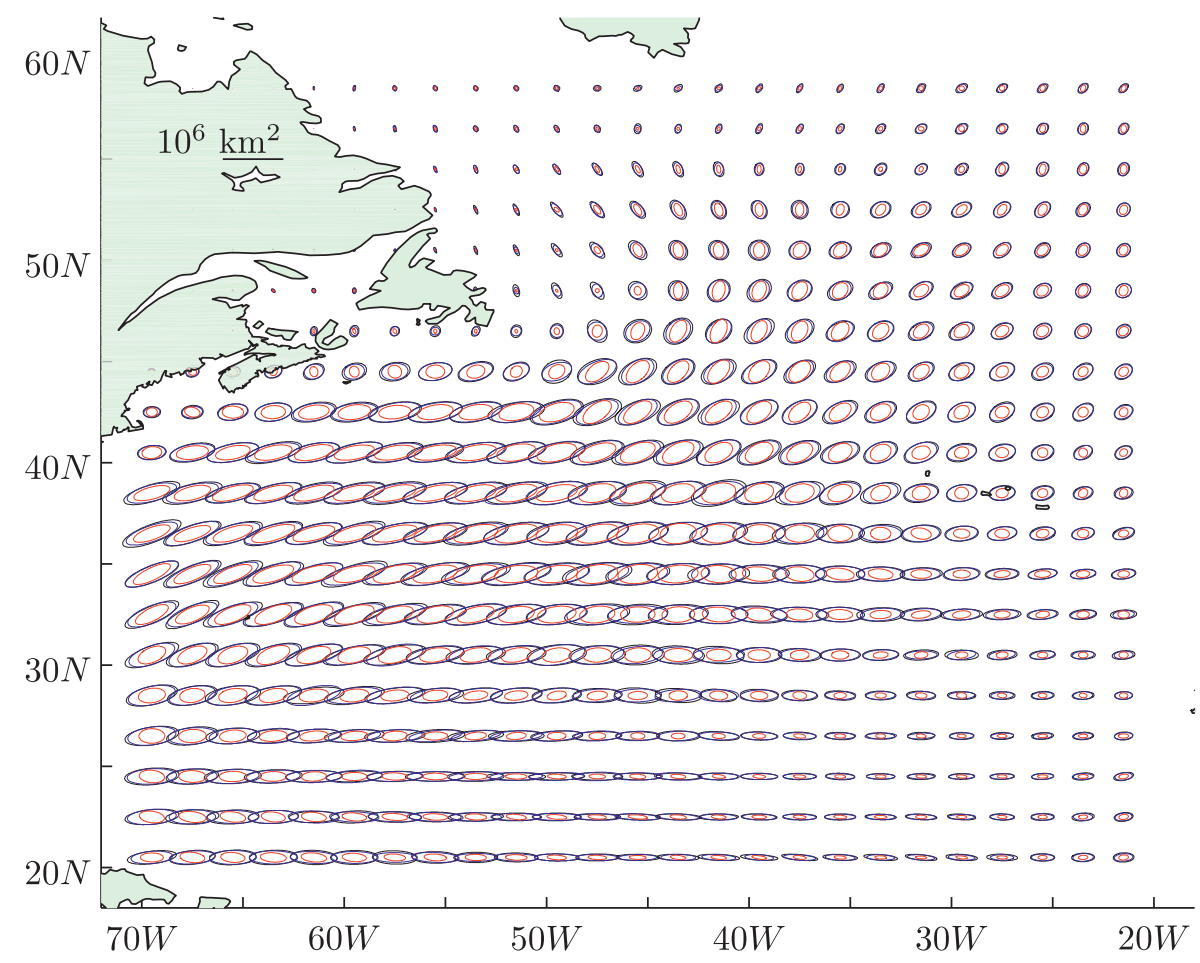

(a)

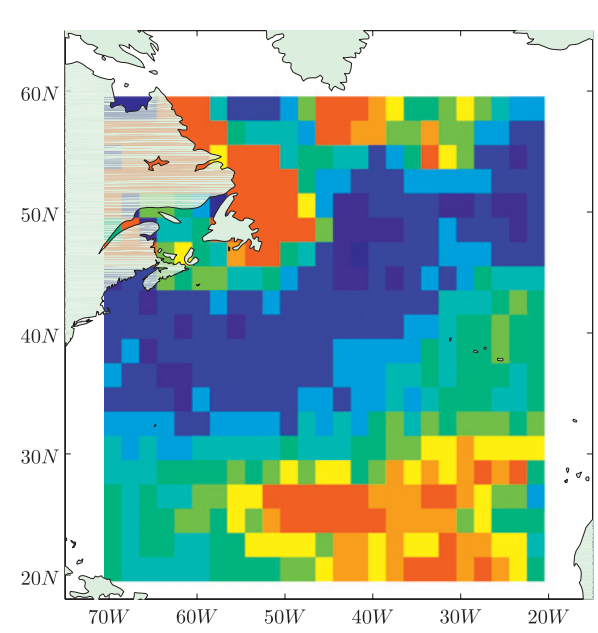

(b)
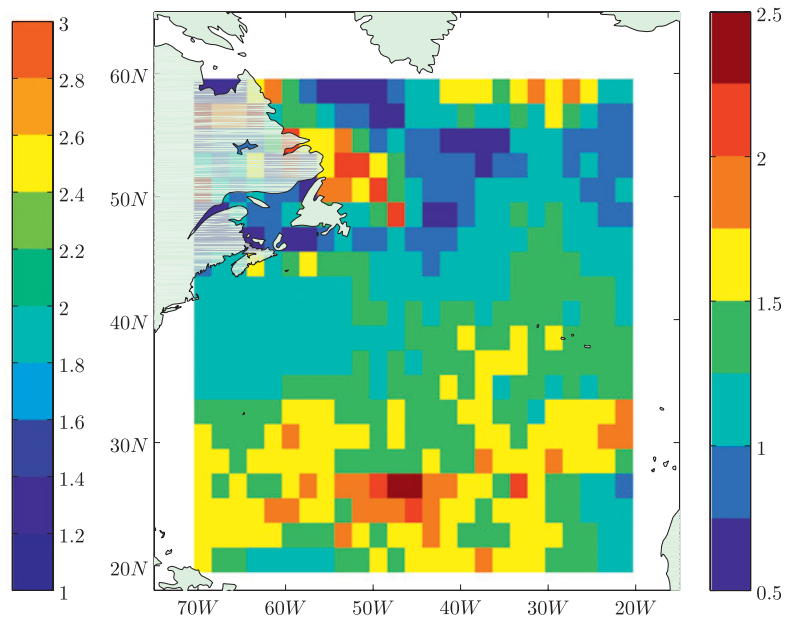

(c)

FIG. 4. (a) EO spreading ellipses for the unfiltered eddy field (black), eddy field with removed annual cycle (blue), and eddy field with removed annual cycle and interannual variability (red). (b) Ratio of the unfiltered and filtered (without annual cycle and interannual variability) $D_{\tau}$. (c) $\left(D_{\tau} / D_{n}\right)_{\text {unfiltered }} /\left(D_{\tau} / D_{n}\right)_{\text {filtered }}$, i.e., ratio of the unfiltered and filtered anisotropy coefficient.

At very short times, compared to the characteristic Eulerian time scale of the eddy field (i.e., the decorrelation time of the velocity at a fixed location), velocity of the particles experiences small changes, therefore, the resulting dispersion grows quadratically with time $\left(D \propto t^{2}\right)$ and the corresponding regime is called ballistic. This is indeed the regime observed on short (order of a few days) time scales in Fig. 5. This is roughly consistent with earlier estimates at midlatitudes (Krauss and Boning 1987; Garraffo et al. 2001) and in Nordic Seas (Andersson et al. 2011). At later stages, when the particles spread further apart from each other, each particle samples many different eddies, and particle trajectories start to resemble random walks. At these times, the dispersion 


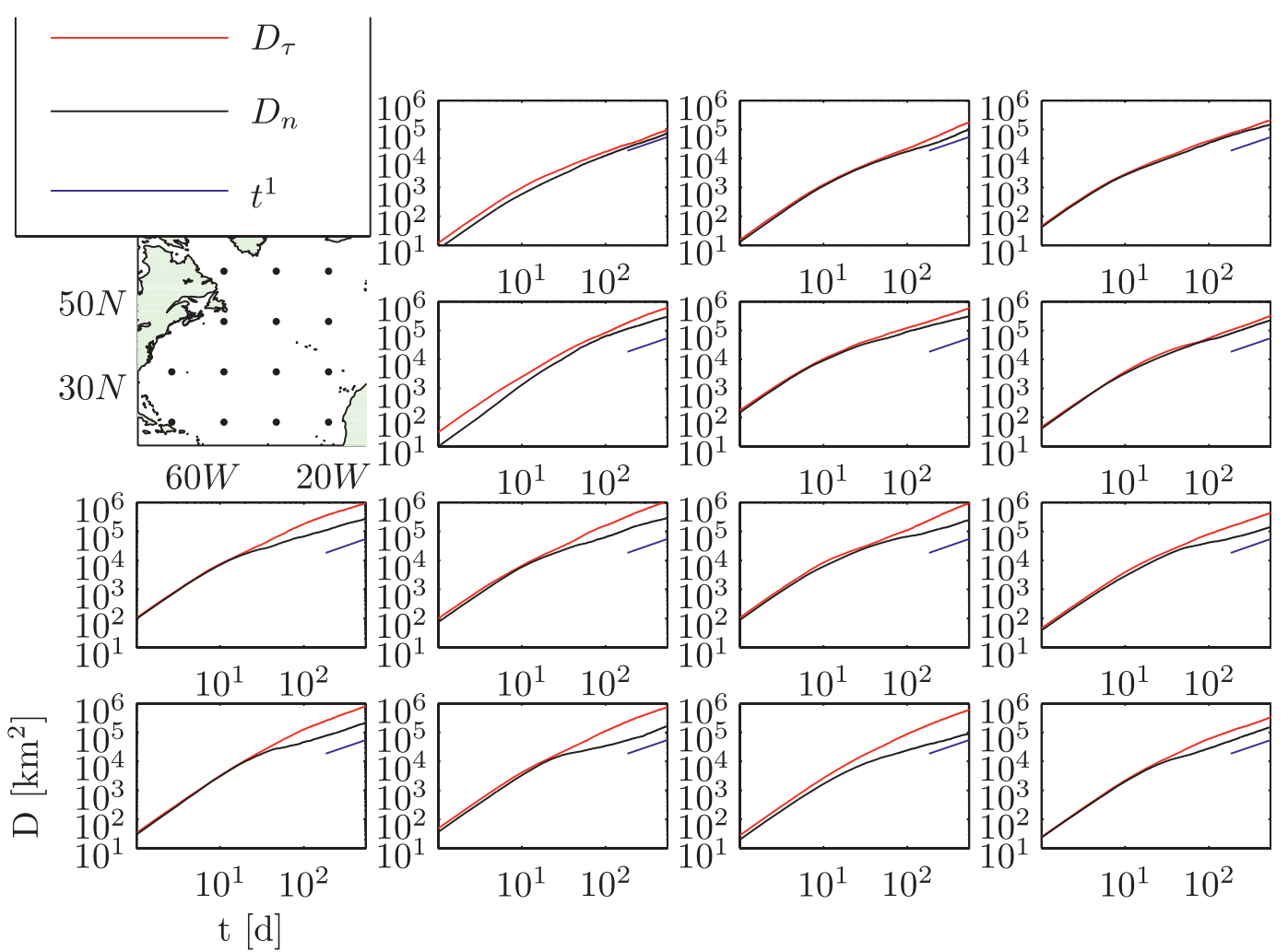

FIG. 5. Plot of $D_{\tau}$ (red) and $D_{n}$ (black) as a function of time for different parts of the North Atlantic for the EO case. To produce each subplot, 100 particles were repeatedly released in a $1^{\circ} \times 1^{\circ}$ bin once per month from October 1992 until July 2007 and were tracked for 1.5 years. Positions of square centers corresponding to each subplot are shown by black dots on the map in the upper left corner. For comparison, diffusive regime $(D \propto t)$ is shown by blue lines.

will reach the diffusive regime $(D \propto t)$ if the Lagrangian velocity decorrelation is complete. We estimated the time required to reach the asymptotic diffusion regime directly from the dispersion curves in Fig. 5 and found that throughout the entire North Atlantic, D starts to grow approximately linearly with time after about half a year. Alternatively, the decorrelation time-scale could be defined as the $e$-folding time of the autocorrelation velocity function (order of a few days in our case), or as the ratio between diffusivity and the velocity variance (as in Vallis 2006) [O(10) days in our case]. Whether the different definitions agree depends on the shape of the velocity autocorrelation. If, for example, the velocity autocorrelation function exhibits the exponential behavior, its integral asymptotes to a constant diffusivity over several $e$-folding times. But if, as in our case, the autocorrelation exhibits significant negative lobes and other deviations from the exponential decay, the asymptotic diffusive regime may occur at much longer timelags. A similar effect, that is, a connection between the existence of the negative lobes in the velocity autocorrelation and the slow approach to the asymptotic diffusive regime, was recently reported by Klocker et al. (2012a,b). Von
Kameke et al. (2011) also observed, in a qualitative agreement with our results, a long delay in the approach to diffusion in a laboratory study of a fluid flow forced by Faraday waves. The relatively long time scale required to reach the diffusive regime in our study is generally consistent with the dominance of eddies with spatial scales on the order of or longer than $200 \mathrm{~km}$. If the spreading rate $K$ is taken to be on the order of or smaller than $10^{4} \mathrm{~m}^{2} \mathrm{~s}^{-1}$ and the eddy spatial scale $L$ - on the order of or larger than $200 \mathrm{~km}$, one can estimate the particle spreading velocity as $K / L$ which would be on the order of or smaller than $0.05 \mathrm{~m} \mathrm{~s}^{-1}$. The time required for particles to move over a distance $L$ is then longer than 1.5 months. If one assumes that, in order to resemble a random walk, particles should sample several eddies, one would get, in a qualitative agreement with Fig. 5, a time scale on the order of or longer than several months, at which a transition to the diffusive regime should occur. More quantitative analysis of the dispersion curves reveals significant deviations from the diffusive regime even on scales longer than half a year. Such deviations are possible if long-time Lagrangian velocity correlations are still present in the flow. To quantify these deviations, we 

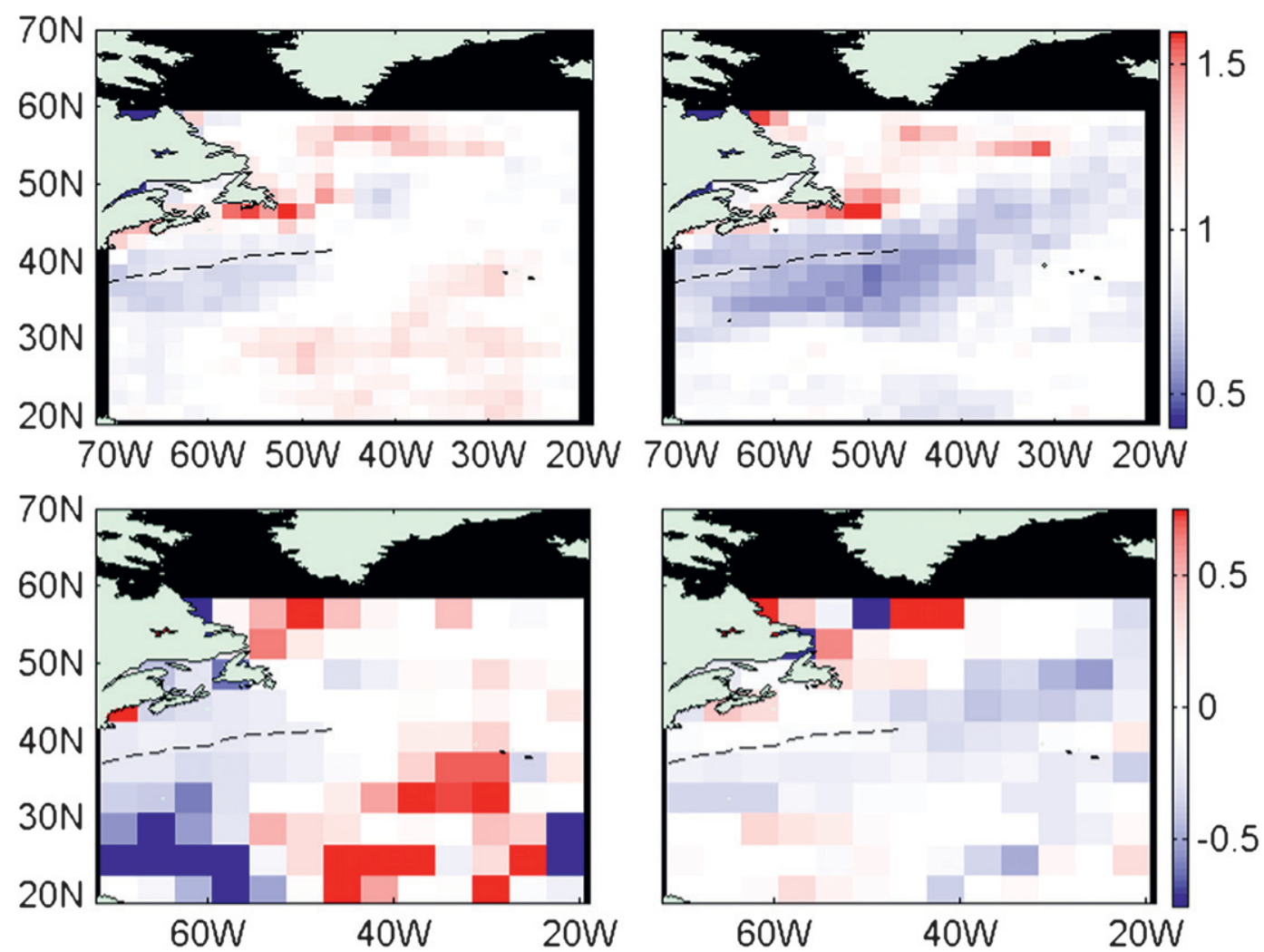

FIG. 6. Eddy-only dispersion exponents, $\alpha_{\tau}$ and $\alpha_{n}$, (top left) along and (top right) across the direction of maximum spreading and the corresponding time-averaged tail (from 0.5 to $1.5 \mathrm{yr}$ ) of the ensemble-averaged Lagrangian velocity autocorrelation (bottom left) along and (bottom right) across the direction of maximum spreading. Black dashed curve is the Gulf Stream core.

fitted $t^{\alpha_{\tau / n}}$ curve to each dispersion curve $D_{\tau / n}(t)$ from 0.5 to $1.5 \mathrm{yr}$ and then plotted the single-particle dispersion exponent, $\alpha_{\tau / n}$, for all $1^{\circ} \times 1^{\circ}$ bins (Fig. 6, top). The two upper subplots of Fig. 6 reveal that the Gulf Stream extension region is characterized by subdiffusive regime $(\alpha<1)$, whereas the subpolar region-by superdiffusive $(\alpha>1)$ regime in both $D_{\tau}$ and $D_{n}$. The central and eastern subtropical gyre shows superdiffusive behavior in $D_{\tau}$ and slightly subdiffusive behavior in $D_{n}$. This pattern is in qualitative agreement with Berloff et al. (2002), who carried out similar analysis of an idealized numerical model of the wind-driven ocean gyres, and with Kamenkovich et al. (2009a), who analyzed a comprehensive general circulation model. The deviation from the diffusive regime, quantified by $\alpha_{\tau}$ and $\alpha_{n}$, is related to the time-averaged tail (from 0.5 to $1.5 \mathrm{yr}$ ) of the ensembleaveraged Lagrangian velocity autocorrelation, which is shown in the two lower panels of Fig. 6: the subdiffusive regime corresponds to the negative time-mean, whereas the superdiffusive regime-to the positive time mean.

One explanation of the subdiffusive behavior of Lagrangian particles in the Gulf Stream region could be that when particles disperse from a region with high
EKE (and high local values of diffusivity) into neighboring regions with lower EKE (and lower local diffusivity), the spreading rate can be expected to decrease with time, leading to a subdiffusive spreading regime in the Lagrangian sense. Denoting the characteristic velocity at which an ensemble of particles is being dispersed in the, for example, $\mathrm{x}$-direction by $U$, the connection between the Lagrangian estimate of diffusivity $K_{L}$ and the Eulerian local diffusivity $K_{E}(x)$ can be written as $d K_{L} /$ $d t=U d K_{E} / d x$ leading to $d K_{L} / d t<0$ when $U d K_{E} / d x<0$. Note that the Lagrangian spreading can be subdiffusive even if the local Eulerian diffusivities are constant in time. This effect can be expected to be more pronounced for the $D_{n}$, because in the Gulf Stream region the EKE ellipses are aligned with the Gulf Stream, so that the EKE gradient is the largest in the $n$ direction. Another explanation could be that the particles are trapped for long times by coherent eddies (Berloff et al. 2002). It is also interesting to note that the super/subdiffusive regime of $D_{\tau}$ and $D_{n}$ in the interior of the subtropical gyre is qualitatively consistent with the presence of the "latent" zonal jets (Maximenko et al. 2005; Berloff et al. 2009, 2011; Kamenkovich et al. 2009a,b) that 


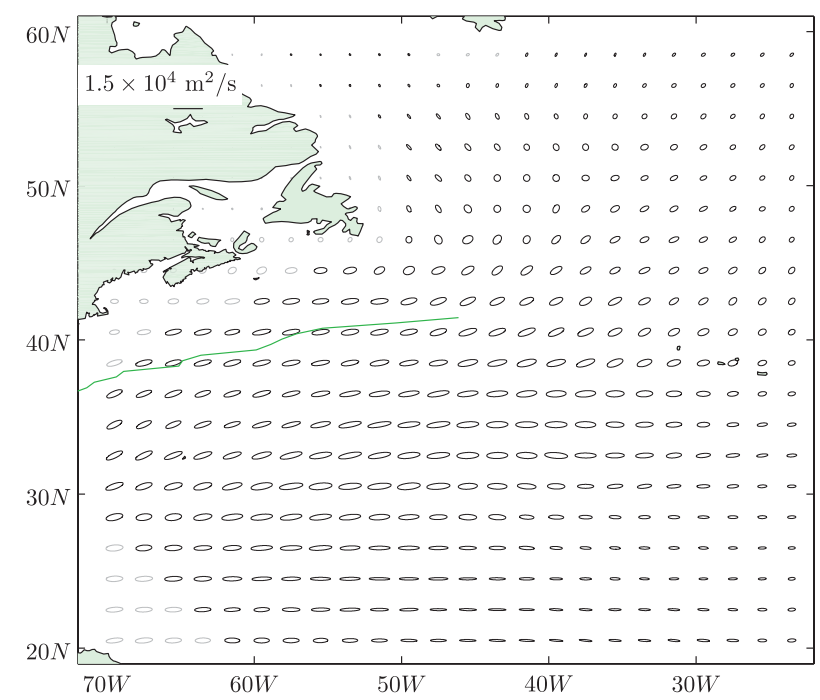

FIG. 7. Eddy-only spreading rate (or diffusivity) ellipses. Particles were released once per month from October 1992 until July 2007 and tracked for 1.5 years. Diffusivity values we estimated over the last year of integration. Gray color indicates bins where more than $40 \%$ of the trajecties are affected by the boundary. Green curve shows the mean Gulf Stream core.

enhance/inhibit the zonal/meridional spreading rates. Although the stationary jets are best seen in the mean zonal velocities averaged over decade(s), they are also present in anomalies averaged over several months (Maximenko et al. 2005).

Despite noticeable local deviations of the spreading from the diffusive regime, spreading rates can still be approximated by fitted time-average diffusivities, which can be used to guide development of diffusion-based parameterizations for non-eddy-resolving models. We estimated the approximate diffusivities along and across the direction of maximum spreading, $K_{\tau}=(1 / 2) d D_{\tau} / d t$ and $K_{n}=(1 / 2) d D_{n} / d t$, respectively, with time derivatives estimated using the best-square linear fits to $D_{\tau}(t)$ and $D_{n}(t)$ over the last year. These "approximate diffusivity" estimates are shown in Fig. 7 in the form of diffusivity ellipses, with slope $\phi$ and the major and minor axes equal to $K_{\tau}$ and $K_{n}$, respectively. As a consistency check, we recomputed diffusivities using integrated velocity correlation method (not shown) and found very similar results.

\section{4. "Integrated-eddy-only" (IEO) and "eddy- following-full-trajectory" (EFFT) simulations based on altimetry}

The analysis in the previous section ignored the influence of the mean velocity, whose effects on the material transport can be significant (Young and Jones 1991;
Smith 2005; Ferrari and Nikurashin 2010; Rypina et al. 2007a, 2011). Even in the complete absence of eddies, the shear and strain of a mean flow will cause neighboring trajectories to separate from each other. In pure shear flow the rate of separation is linear in time; in a flow with pure strain the separation can be exponential with time. Estimates of eddy dispersion must take care to subtract out these effects. For instance, if the diffusivities in (2) are estimated from the "full" particle trajectories (particles advected by the full flow), the resulting values represent a combined dispersion by the mean currents and eddies and thus cannot be used for parameterizing the eddy-induced transfer (which should only represent eddies, not the mean flow). On the other hand, the EO calculation, where the mean is completely removed at the outset, ignores the fact that in the full flow a particle is carried through the eddy field in different geographical regions and is moving faster through some parts of the eddy field and slower through the others compared to the EO case. Thus, the transport properties of the eddy field in the presence of the mean advection, that is, from the point of view of a particle advected by the full flow, can differ from the properties of the same eddy field in the EO sense. The goal of this section is to investigate this effect, and we use two ways to achieve this. The first one, which we refer to as the "integrated-eddy-only" or IEO method, accounts for the variations of diffusivity encountered as one moves through regions of different local diffusivity along a mean trajectory, but assumes that local diffusivities are the same as in the EO case. The second method, which we refer to as the "eddy-following-full-trajectory" or EFFT method, accounts both for the advection to different geographical regions and for the changes in local eddy diffusivities. In the EFFT calculation, the mean displacement is subtracted from the total displacement over each time step. This removes the effects of mean shear and strain in a continuous manner, leaving a dispersion due only to eddies. Both methods are explained in more detail below.

Consider a flow consisting of the mean and eddying components, where the mean component is known, but the eddying component is not. Our objective is to quantify the diffusive effects of the eddying component. This task is highly relevant to the parameterization of transient eddies in low-resolution models that severely underestimate variability in the flow, but reproduce the mean currents fairly well. The mean currents will carry particles (or a tracer patch) through regions with different local diffusivities, and a simple way to account for the resulting particle dispersion is to integrate the local EO dispersion rates along the mean trajectory $\left(x_{\mathrm{tr}}(t)\right.$, $\left.y_{\mathrm{tr}}(t)\right)$ as follows: 


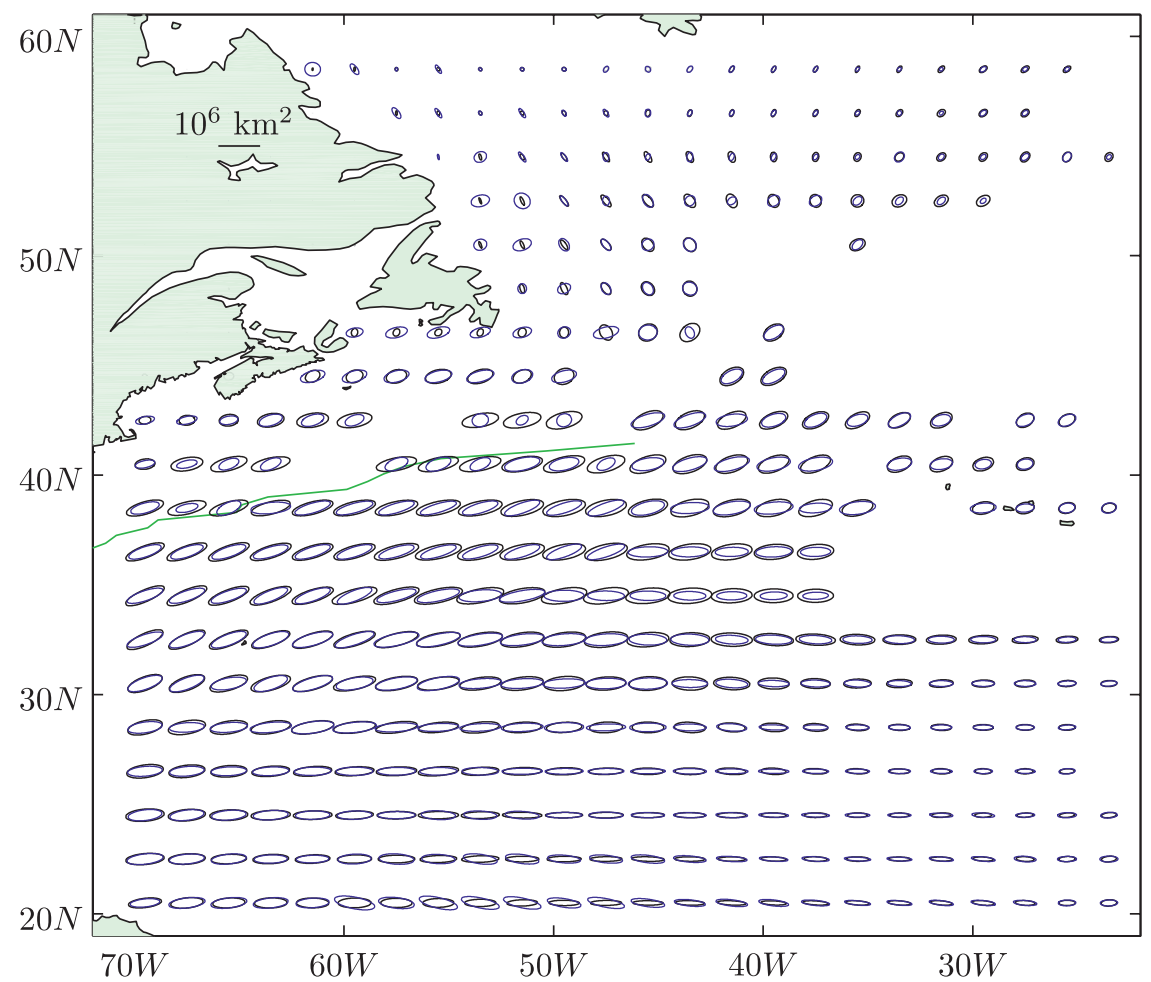

FIG. 8. Spreading ellipses for the integrated-eddy-only case (in blue), computed by integrating the eddy-only diffusivities (Fig. 7) along the mean trajectories. For comparison, the eddy-only ellipses are shown in black (same as in Fig. 2). Areas from where the mean trajectories venture outside of our domain are left blank. Green curve shows the mean Gulf Stream core.

$$
D=2 \int_{t_{\mathrm{st}}}^{t_{\mathrm{end}}} K\left[x_{\mathrm{tr}}(t), y_{\mathrm{tr}}(t)\right] d t .
$$

We will refer to this situation as the "integrated-eddyonly" or IEO case, which is short for "integrating-eddyonly-diffusivities-following-mean-trajectories." In the IEO analysis (Fig. 8), the effects of the mean advection are clearly seen in locations, from where particles are advected into regions with different diffusivities. This effect is most pronounced for trajectories that originate around and slightly north of the mean Gulf Stream path (characterized by large values of $K$ ) and then cross into the western subpolar region (characterized by small values of $K$ ). There, the IEO ellipses (blue) differ substantially from the corresponding EO (black) ellipses. In regions where trajectories are not advected far or where diffusivities are more homogeneous, the EO and IEO ellipses are similar to each other.

The above analysis makes assumption that the EO estimates of $K$ are accurate, and the only effect of the mean advection is to integrate $K$ along the mean-flow trajectories. However, full-flow trajectories will differ from the mean-flow trajectories and, as discussed above, in addition to being advected into different geographical regions, the particles will experience the eddy field in a different manner compared to the EO case. To investigate these effects, we calculated the "eddy-following-full-trajectory" (or EFFT) spreading ellipses (Fig. 9), which characterize the cumulative effect of the eddy field that the particle experiences as it moves through the full flow. To extract the eddy-induced dispersion from the full-flow dispersion, on each time step, we corrected for the mean advection by subtracting the displacement due to the mean field from the total displacement following the trajectory. The resulting eddy-induced displacement vectors are then cumulatively added together. The resulting "pseudo-trajectory" due to the eddy field is then analyzed in the same way as the EO trajectories (section 3). We estimate the dispersion, $D_{x / y / x y}$, and the spreading rate, $K_{x / y / x y}$, using (1), (2), and (5), and then calculate the angle of maximum spreading, $\phi$, thus obtaining the EFFT spreading ellipses. In areas where the mean circulation is much weaker than the eddy field, the EFFT and the EO dispersions are expected to be similar. In contrast, where the mean and eddy fields become comparable in magnitude, the structure of the EFFT ellipses depends on the details of the flow, and can be substantially different from the EO case. 


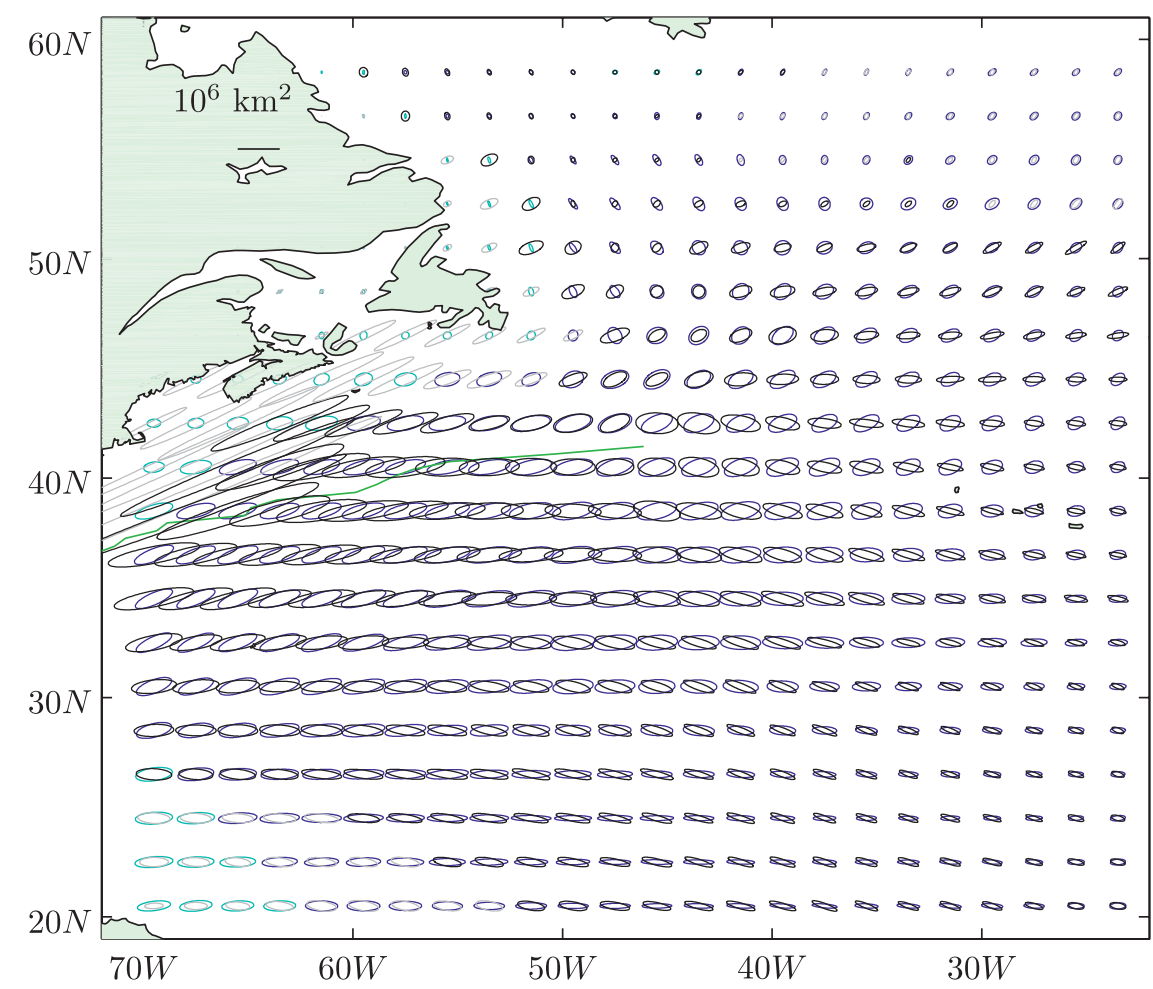

FIG. 9. Spreading ellipses for the eddy-following-full-trajectory case. Particles were released once per month from October 1992 until July 2007 and tracked for 1.5 years using altimetric velocities; the spreading ellipses were calculated at the end of 1.5 years. Gray color corresponds to bins where more than $40 \%$ of the trajecties are affected by the boundary. For comparison, the black and gray eddy-following-full-trajectory ellipses are superimposed on the blue and cyan eddy-only ellipses (same as the black and gray ellipses, respectively, in Fig. 2). Green curve shows the mean Gulf Stream core.

Regardless of how the effects of the mean advection are accounted for, in the presence of the mean circulation the eddy-induced transport remains highly anisotropic with the spatially averaged anisotropy coefficient $D_{\tau} / D_{n} \sim 5$ for the subtropical and $\sim 2.5$ for the subpolar gyre (Fig. 9). However, the effects of the mean advection are substantial in several regions. The most noticeable difference between the EFFT and EO ellipses is in the western North Atlantic over the Georges Banks, where the mean and eddy fields are both strong and similar in magnitude. In this region, the EFFT ellipses (Fig. 9) are much larger than the EO ellipses (Fig. 2), and the former spreading is more anisotropic. This difference can be attributed to the fact that, in the EO simulations, the particle trajectories tend to disperse away from regions with stronger eddies, whereas in the EFFT case, the mean circulation "keeps" trajectories in these regions for longer time, thus, resulting in larger dispersion values. Throughout the rest of the subtropical gyre, the magnitudes and shapes of the ellipses in the EO and EFFT cases are similar. The tilts of the ellipses in the two cases are particularly close to each other in the western part of the subtropical gyre, but are noticeably different further to the east. There, the EO ellipses are nearly zonal but the EFFT ellipses have slightly negative tilts. Note also, that a significantly larger portion of the floats is affected by the coast in the EFFT case than in the EO simulation, because of the larger float displacements and longer float trajectories.

Like in the EO case, the EFFT dispersion (Fig. 10) is initially superdiffusive, and it becomes more diffusive on time scales longer than about half a year. The spatial structures of the EFFT $\alpha_{\tau}$ and $\alpha_{n}$ and the associated Lagrangian velocity autocorrelation functions are shown in Fig. 11. As in the EO case, the Gulf Stream extension region is characterized by the subdiffusive regime, the subpolar gyre by the superdiffusive regime, and the subtropical gyre by the super/subdiffusion in $D_{\tau} / D_{n}$, respectively. The major difference is near boundaries in the northwestern part of the domain, where the EFFT dispersion exponents are larger than the EO values. The deviations from the diffusive regime are also well correlated with the anomalies of the Lagrangian autocorrelation functions. Despite the deviations from the diffusive regime, we have converted the EFFT dispersion into the diffusivities using 


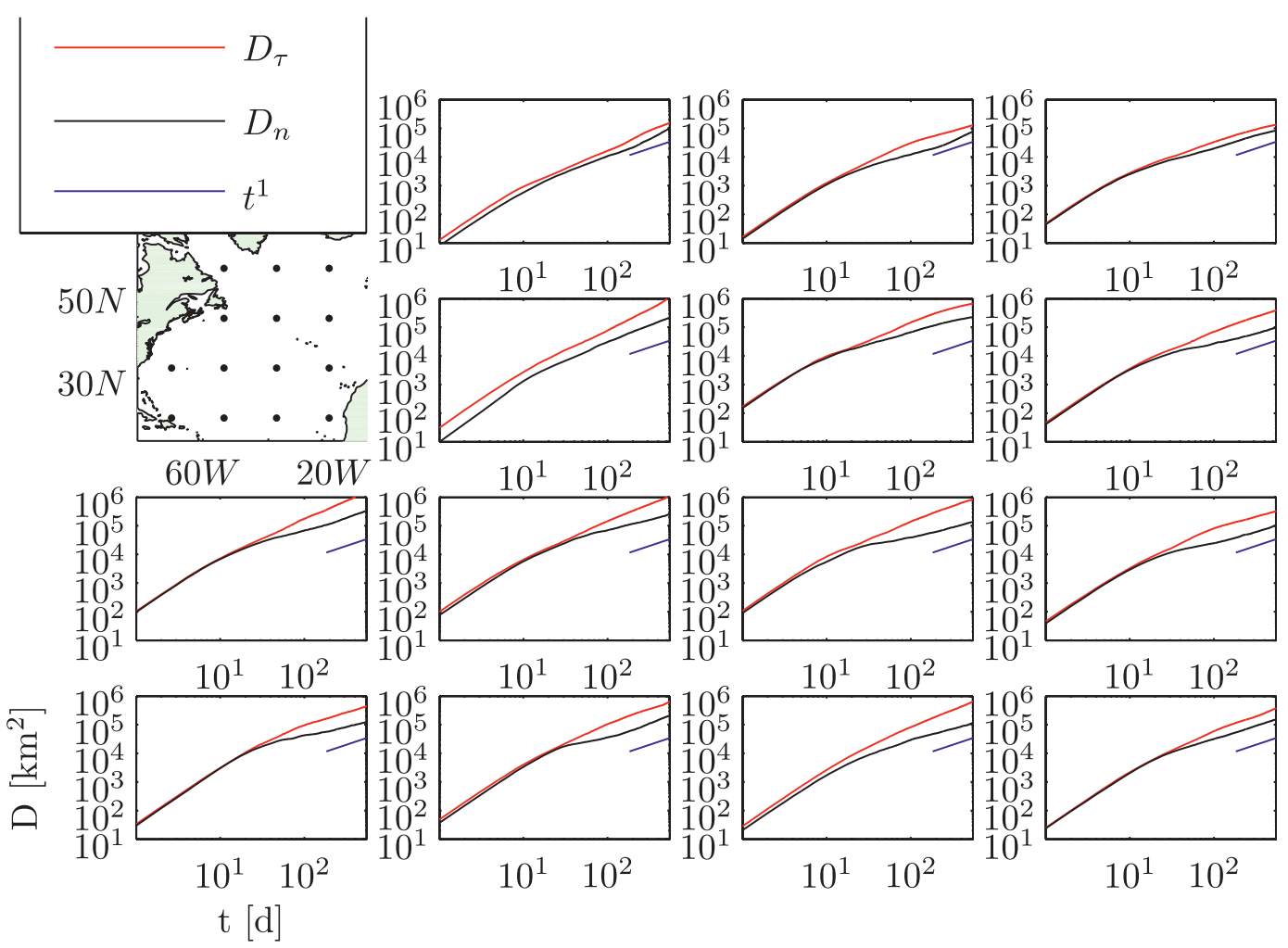

FIG. 10. As in Fig. 5, but for the eddy-following-full-trajectory simulations.

Eq. (2), and the resulting EFFT diffusivity ellipses are shown in Fig. 12.

We shall conclude by saying that, since the mean advection can significantly alter eddy diffusivities, it is precisely these altered diffusivities that one would need to use in models to parameterize the effects of eddies in noneddy-resolving simulations. We believe that the EFFT estimates of $K_{\tau}$ and $K_{n}$ are arguably more relevant for this task than the EO estimates. This is further supported by our analysis of the particle dispersion in an idealized jet (see appendix). However, this issue needs to be thoroughly tested in simulations with tracer (dye) releases before one could say with certainty whether either the EFFT or EO estimates give realistic tracer distributions.

\section{Analysis of drifter trajectories}

\section{a. Drifter-based spreading ellipses}

To compare the results obtained from simulated Lagrangian particles against real oceanic drifters, we carried out dispersion analysis of the satellite-tracked surfacedrifting buoy trajectories from the Global Drifter Program (GDP) (http://www.aoml.noaa.gov/phod/dac/index. php). Note that, unlike simulated trajectories in the previous sections, these trajectories are due to both geostrophic and ageostrophic velocities and include all spatial scales. Since GDP drifters are advected by the real velocity field that includes both the mean circulation and the eddies, it is extremely difficult to use their trajectories to estimate EO spreading ellipses, but it is possible to use these trajectories for estimating the EFFT ellipses. The mean field used in this calculation (shown in Fig. 13), was estimated by binning velocities of the GDP drifters into $1^{\circ} \times 1^{\circ}$ bins and by averaging them over the observation time interval. Note that this technique is based entirely on the GDP dataset. A downside is in possible sampling biases in our estimates of the mean velocities, due to insufficient number and uneven distribution of the drifters in time and space.

Although the GDP dataset includes $\sim 4000$ trajectories (or continuous segments of trajectories) in the North Atlantic, from 1972 to 2009, it is still insufficient to match the statistics used in sections 3 and 4 , and to group trajectories into $1^{\circ} \times 1^{\circ}$ bins according to their deployment locations. It is possible, however, to calculate the spreading ellipses by computing dispersion values for fans of trajectory segments that come out of each bin. In other words, we combine segments of trajectories that pass through a given bin from the time of exit and forward. To improve the statistics, we also add trajectory segments from the time of exit from the bin and backward in time, that is, we add a fan of trajectory segments 

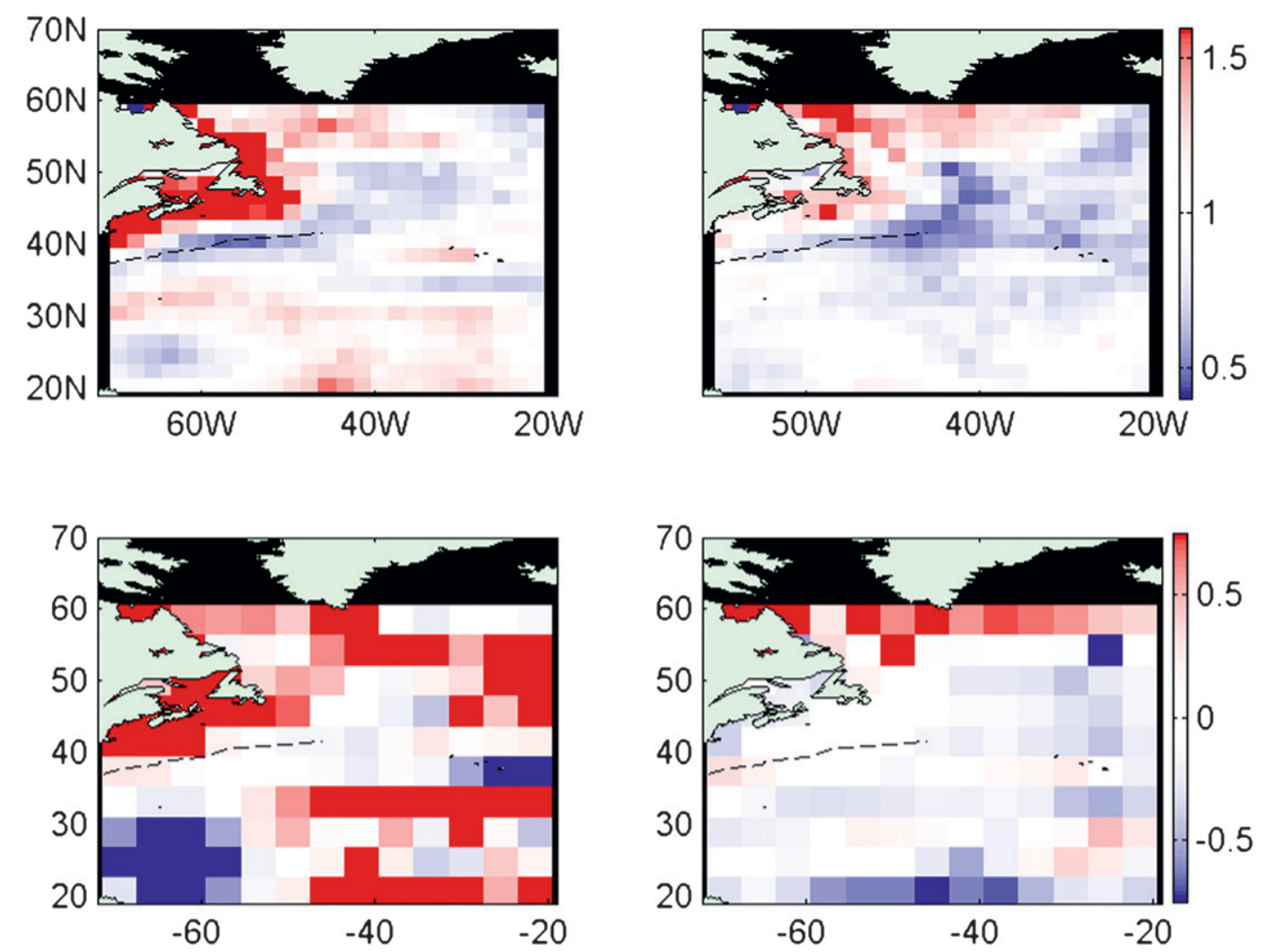

FIG. 11. As in Fig. 6, but for the eddy-following-full-trajectory simulations.

that enter the bin. Finally, since trajectory segments are of different length, it is not possible to carry out analysis over the same time-interval in all the bins (as in Fig. 12), therefore we used different time intervals for different bins, and bins containing less than 50 trajectory segments were not considered. Because of these limitations, the GDP-based dispersion estimates should be considered with caution (rather than assumed to be the "ground truth").

Several aspects of the EFFT ellipses from the GDP dataset (Fig. 14) are qualitatively similar to those of the altimetry-based ellipses (Fig. 12). First, both sets of ellipses are highly anisotropic (with the domain-averaged anisotropy coefficient $R_{K} \sim 3.1$ for the GDP case). Second, both sets of ellipses are predominantly zonal in the central subtropical gyre, with slightly positive tilt in the Gulf Stream region and its eastward extension. Third, the fitted diffusivity values have maximum in the western part of the North Atlantic over the Georges Banks (although the GDP estimates in this region are about twice smaller). The ranges of the GDP-based diffusivity estimates are $\left(0.1 \leq K_{\tau} \leq 3.4\right) \times 10^{4} \mathrm{~m}^{2} \mathrm{~s}^{-1}$ and $\left(0.05 \leq K_{n} \leq\right.$ 1) $\times 10^{4} \mathrm{~m}^{2} \mathrm{~s}^{-1}$ with $\left\langle K_{\tau}\right\rangle_{\text {mean }}=1.3 \times 10^{4} \mathrm{~m}^{2} \mathrm{~s}^{-1}$ and $\left\langle K_{n}\right\rangle_{\text {mean }}=0.4 \times 10^{4} \mathrm{~m}^{2} \mathrm{~s}^{-1}$, where $\langle\cdot\rangle_{\text {mean }}$ denotes the domain-averaged value. The corresponding altimetrybased estimates are $\left(0.06 \leq K_{\tau} \leq 12\right) \times 10^{4} \mathrm{~m}^{2} \mathrm{~s}^{-1}$ and $\left(0.02 \leq K_{n} \leq 0.5\right) \times 10^{4} \mathrm{~m}^{2} \mathrm{~s}^{-1}$ with $\left\langle K_{\tau}\right\rangle_{\text {mean }}=0.8 \times$ $10^{4} \mathrm{~m}^{2} \mathrm{~s}^{-1}$ and $\left\langle K_{n}\right\rangle_{\text {mean }}=0.2 \times 10^{4} \mathrm{~m}^{2} \mathrm{~s}^{-1}$. The quantitative agreement between the GDP and altimetric estimates is the best in the western subtropical gyre south of the Gulf Stream, where the GDP data coverage is also very good. However, several regions exhibit noticeable differences between two estimates. First, there is a mismatch between the ellipse tilts in the southeastern North Atlantic, where tilts are positive for the GDP drifters but negative for the simulated drifters. This difference may be partially due to the local bias in the estimated mean circulation: the GDP-based mean velocities are stronger than the altimetric mean velocities. Note that this region generally has poor GDP data coverage and is adjacent to the part of the domain with particularly low data coverage (shown by blank in the figure). Note also that some of the GDP data in this area date back before 1992, but the altimetric estimates are for 1992-2009, so any possible differences in the mean circulation and eddy statistics before and after 1992 might contribute to the difference between drifter-based and altimetric estimates. The second main difference between Figs. 14 and 12 is in slightly larger mean diffusivity values for the GDP case, and this is more so in the subpolar rather than subtropical region. A possible explanation is insufficient spatial resolution 


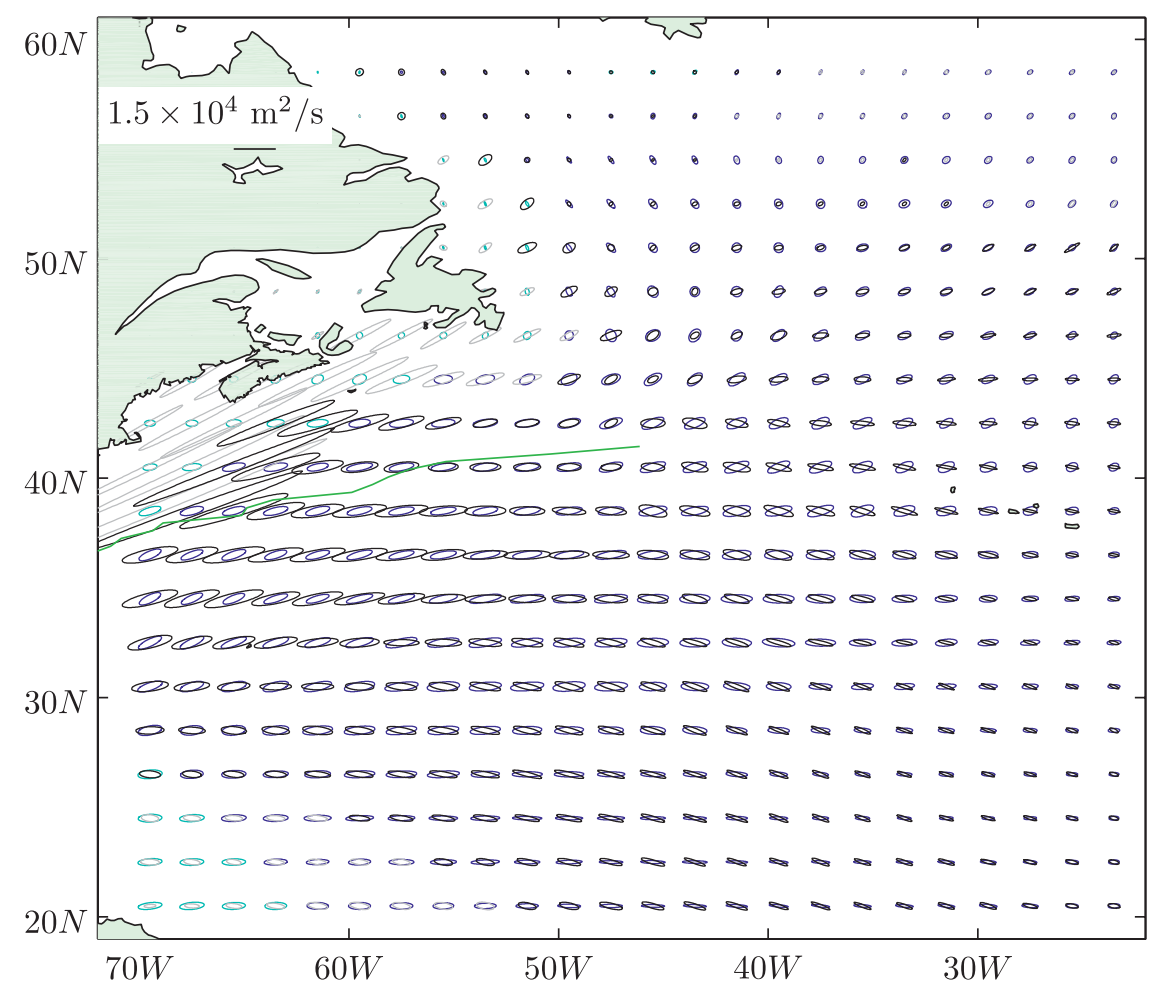

FIG. 12. As in Fig. 7, but for the eddy-following-full-trajectory simulations. For comparison, the eddy-only diffusivity ellipses are shown in blue and cyan. Green curve shows the mean Gulf Stream core.

of the altimetric velocity fields, that is more pronounced in the subpolar region characterized by smaller eddies (due to a smaller first Rossby radius of deformation). Simulations of idealized geostrophic turbulence (Kamenkovich et al. 2012, manuscript submitted to J. Phys. Oceanogr.) suggest that underresolved velocity (with spatial grid resolution coarser than 16 first Rossby deformation radii) results in substantial (more than $25 \%$ ) decrease of both the eddy

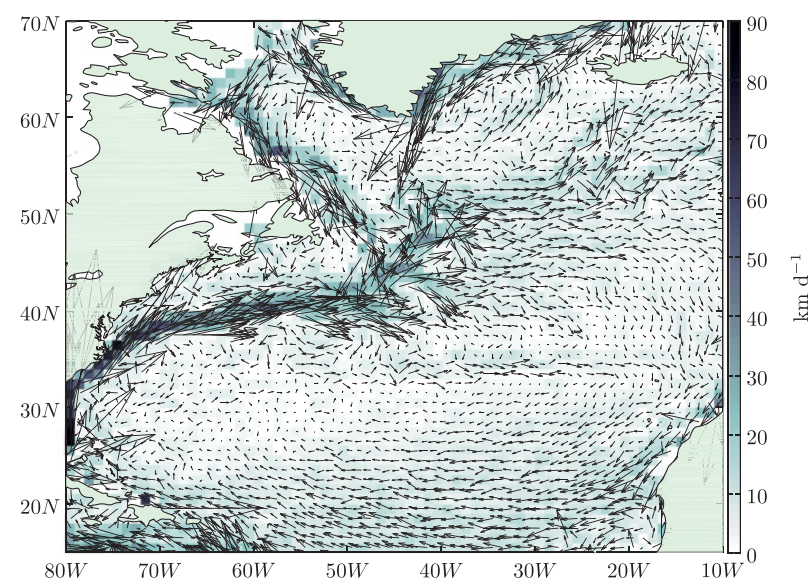

FIG. 13. Mean drifter-based circulation in the North Atlantic. diffusivity and the anisotropy coefficient. These resolution dependencies, however, are to be evaluated in more realistic flow simulations.

The GDP dispersion exponents, $\alpha_{\tau}$ and $\alpha_{n}$ (Fig. 15) are in good agreement with the EFFT results: the Gulf Stream

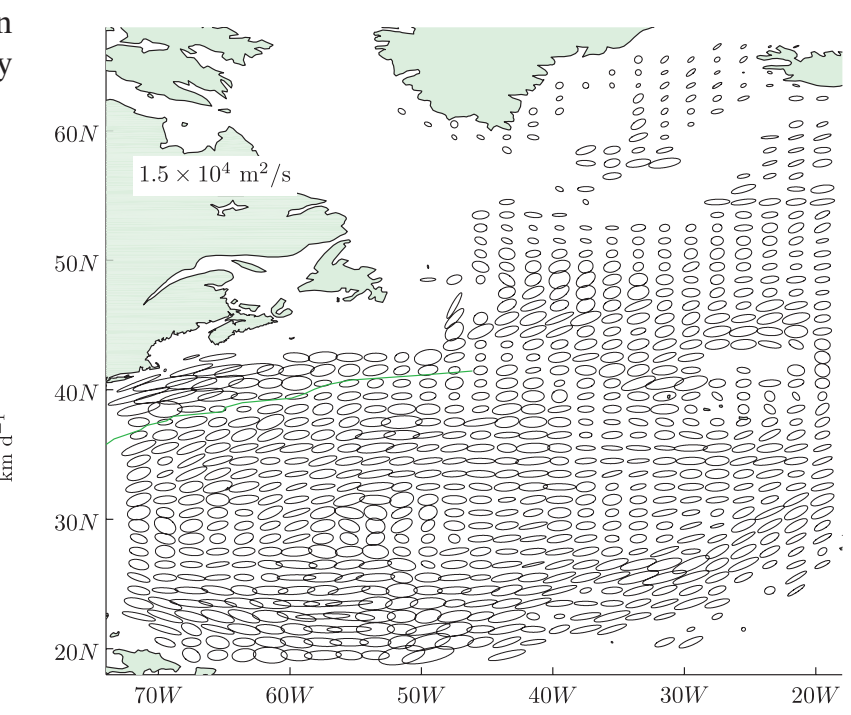

FIG. 14. Eddy-following-full-trajectory diffusivity ellipses found from the GDP dataset. Green curve shows the mean Gulf Stream core. 


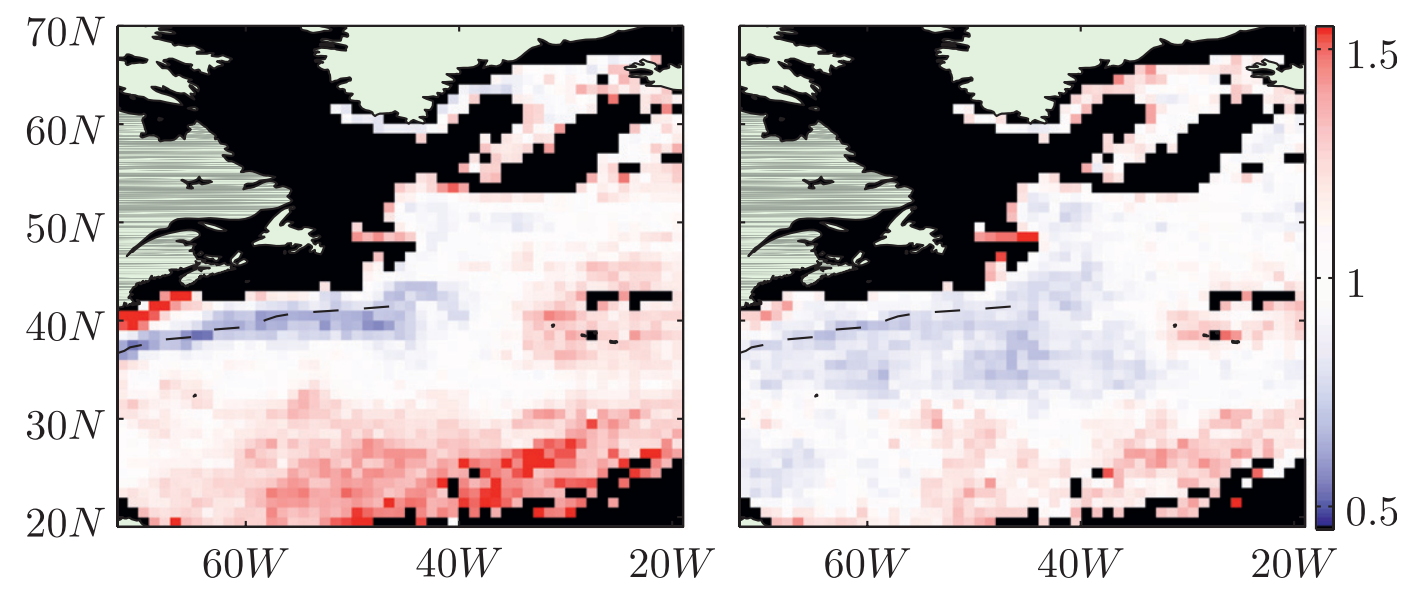

FIG. 15. Dispersion exponents, $\alpha_{\tau}$ and $\alpha_{n}$, along and across the direction of maximum spreading (left and right, respectively), computed from the GDP dataset. Bins with less than 50 trajectories are masked by black.

extension contains the subdiffusive regime for both $D_{n}$ and $D_{\tau}$; the subpolar region contains the superdiffusive regime in both $D_{n}$ and $D_{\tau}$; and the southern subtropical region has highly superdiffusive behavior in $D_{\tau}$ and slightly superdiffusive behavior in $D_{n}$. The spreading regime for $D_{\tau}$ in the latter region is arguably closer to being ballistic $\left(\propto t^{2}\right)$ rather than diffusive $(\propto t)$, suggesting that the local bias in the GDP mean circulation estimate may be significant. Another factor, possibly leading to larger dispersion exponents in this area, is a relatively small time interval used for the diffusivity estimates. Overall, despite some regional differences, there is qualitative agreement between the GDP and altimetric results.

\section{b. Influence of the Ekman velocity}

Another possible source of discrepancy between the GDP and altimetric results is the absence of the nearsurface ageostrophic velocities, including Ekman velocities, in the latter but their presence in the former dataset. We explored the influence of the Ekman velocities by adding them to the altimetric velocities and recomputing the spreading rate ellipses. The Ekman velocity $\left(U_{\mathrm{ek}}, V_{\mathrm{ek}}\right)$ used in our calculations was estimated using NASA's Quick Scatterometer (QuikSCAT) wind stresses and the Ralph and Niiler (Ralph and Niiler 1999) formula,

$$
u_{\mathrm{ek}}+i v_{\mathrm{ek}}=\frac{\beta e^{-i \theta}}{\sqrt{f \rho}} \frac{\tau_{x}+i \tau_{y}}{\sqrt{|\boldsymbol{\tau}|}},
$$

where $\tau$ is the wind stress at $10 \mathrm{~m}$ height, $\rho=1027 \mathrm{~kg} \mathrm{~m}^{-3}$ is the water density, $f$ is the Coriolis parameter, $\theta=$ $55^{\circ}$ is the rotation angle of the Ekman current, and $\beta=$ $0.065 \mathrm{~s}^{-1 / 2}$ is dimensional constant. The resulting mean and the standard-deviation values of the Ekman velocities are shown in Fig. 16.
The comparison between either the standard ("noEkman") or the Ekman-inclusive cases and the GDP case (Fig. 17) suggests that the Ekman-inclusive ellipses are slightly more zonal and isotropic than the standard ellipses. The basin-average values for the Ekman-inclusive case are $\left\langle K_{\tau}\right\rangle_{\text {mean }}=0.7 \times 10^{4} \mathrm{~m}^{2} \mathrm{~s}^{-1}$ and $\left\langle K_{n}\right\rangle_{\text {mean }}=0.35 \times$ $10^{4} \mathrm{~m}^{2} \mathrm{~s}^{-1}$. The largest differences between the Ekmaninclusive and standard cases are seen in the northwestern corner of the subtropical gyre, in-shore from the Gulf Stream extension current. There, with the inclusion of the Ekman velocities, the ellipses are smaller, less anisotropic, and more zonal. In this region, the main effect of the Ekman velocities is additional mostly meridional advection, which moves particles across the Gulf Stream axis. This effect reduces the impact of anisotropic, along-stream eddy spreading. Also, the Ekman velocities result in the increase of the diffusivity values in the subpolar gyre, which is also characterized by meridional Ekman advection. There, it is plausible that the Ekman advection enhances otherwise sluggish dispersion by moving particles over a larger area of strongly meridionally sheared flow, and causing the effect similar to shear dispersion (Young and Jones 1991). The Ekman-inclusive estimates are still smaller than the GDP ones, especially in the subpolar gyre and in the eastern part of the subtropical gyre. Our hypothesis is that these differences are caused by the insufficient spatial resolution of the altimetric velocities rather than by the ageostrophic component of the flow. Note, however, that we cannot examine the importance of submesoscale currents, which can be potentially important.

\section{Discussion and conclusions}

This study examines the spatial distribution and anisotropic properties of the near-surface dispersion of 


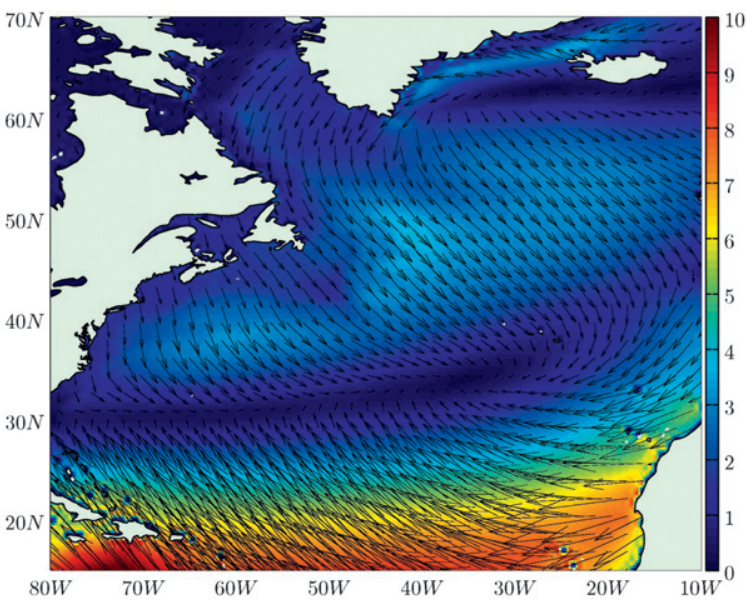

(a)

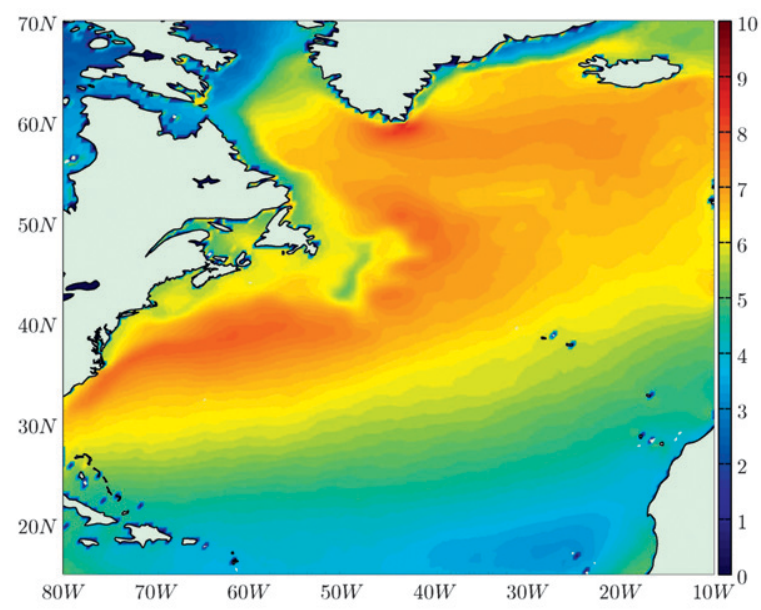

(b)

FIG. 16. (a) Time-averaged Ekman velocity. (b) Square root of the time-averaged Ekman perturbation velocity squared.

particles by transient eddies in the North Atlantic, as estimated from two independent datasets of Lagrangian trajectories: one simulated from the sea surface height altimetry and another one derived from the drifters. Our altimetry-based analysis focuses on intermediate-scale $(>150 \mathrm{~km})$ geostrophic component of the flow, which is resolved by the altimetry. Studies of Kamenkovich et al. (2012, manuscript submitted to J. Phys. Oceanogr.) and Keating et al. (2011) suggest that eddies on these scales play the dominant role in the particle dispersion by geostrophic currents. The results of our study are presented in terms of the "spreading ellipses"- a convenient way of portraying properties of the particle dispersion. The tilt of the ellipse and the ratio between the major and minor axes show the preferred direction and the degree of anisotropy of particle spreading. The results consistently demonstrate strongly anisotropic material transport. In its most straightforward definition, the term "eddy field" refers to all deviations from the long-term mean flow and includes the climatological annual cycle, interannual variability, and subannual (scales shorter than one year) variations. We have investigated the contribution of the annual cycle and interannual variability on both the Eulerian structure of the eddy field (i.e., on the EKE ellipses) and on the Lagrangian particle spreading. Three conclusions were drawn from this analysis. First, the influence of the annual cycle was found negligible. Second, the structure of the spreading ellipses was found significantly different, and thus cannot be inferred directly, from the EKE ellipses. And third, even after the removal of the annual cycle and interannual variability, the spreading ellipses are still anisotropic, suggesting that the subannual variability alone was still causing anisotropy.

We found significant spatial inhomogeneity and strong anisotropy of the particle dispersion. Spreading ellipses are larger and more anisotropic in the subtropical than in the subpolar gyre, in agreement with previous model-based estimates by Kamenkovich et al. (2009a). Ellipses are approximately zonal in most of the domain, with the exception of the northwestern subtropical gyre and the vicinity of the Gulf Stream eastward extension, where the ellipses nearly align with the current axis. The spreading rates are also the largest over there, consistent with the local maximum of the eddy kinetic energy. These properties are found in both datasets, despite some regional differences in the local spreading rates. Our study suggests that this significant anisotropy is a fundamental property of oceanic flows.

The character of the particle spreading changes with time. Ballistic spreading is observed on short, order of a few days, time-scales, which is consistent with the $e$-folding time of the Lagrangian velocity autocorrelation function. The integral Lagrangian time-scale, defined as a ratio between the diffusivity and the velocity variance, is slightly longer $[O(10)$ days]. The time required to reach the diffusive regime is, however, much longer $[O(100)$ days] due to the significant deviations of the velocity autocorrelation function from the exponential shape.

The mean currents can significantly influence the effective eddy-induced particle spreading by moving particles to different geographical regions and by altering the manner in which the particles sample the eddy field. For example, in the presence of the mean currents, the particle can spend longer time in some regions and shorter time in the others compared to the no-mean case. We examined two ways to account for this effect. In one method, referred to as the "integrated-eddy-only" or IEO approach, the particle spreading is calculated by integrating local EO dispersion rates following the mean trajectory. In the second approach, referred to as the "eddy-following-full-trajectory" or EFFT case, we isolate the contribution of the eddy field following full 


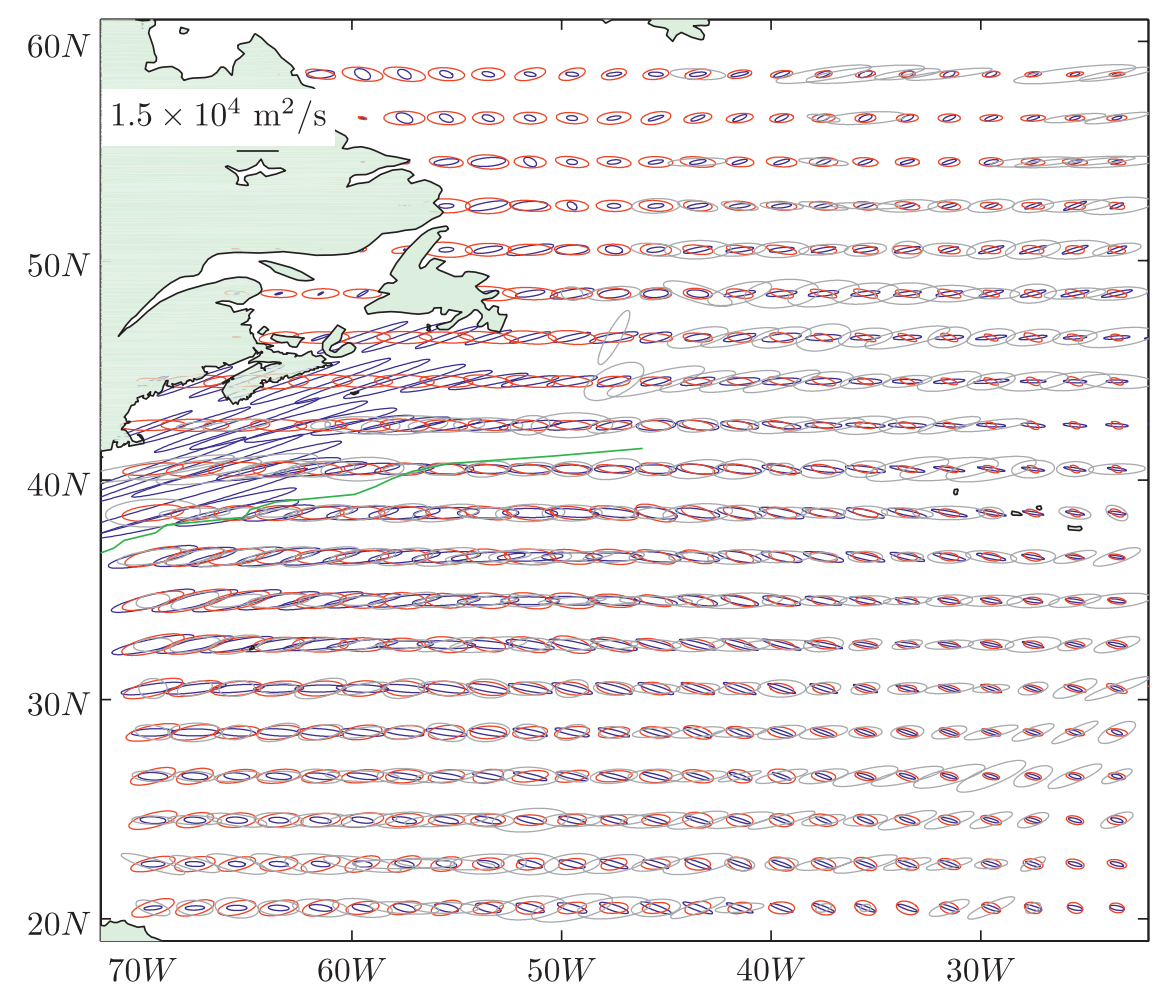

FIG. 17. Ekman-exclusive (blue), Ekman-inclusive (red) and GDP (gray) spreading ellipses. Green curve shows the mean Gulf Stream core.

(mean plus eddy-driven) trajectories by subtracting the mean displacement from the full displacement at each time step following each trajectory, and then estimate the dispersion of the "pseudo-trajectories" constructed as a cumulative sum of the eddy-induced displacements. The resulting eddy-induced particle spreading is different in the two cases in many parts of the domain, most notably, in the northwestern subtropical gyre in the vicinity of the Gulf Stream extension, suggesting that diffusivity is sensitive to how these effects of the mean advection are accounted for.

Processes defining the preferred direction and degree of anisotropy of the eddy-induced particle spreading remain unclear. One plausible explanation is that the spreading takes place primarily along mean potential vorticity (PV) contours (O'Dwyer et al. 2000). We were not able to confirm this hypothesis from our analysis. Although the eddy-induced spreading ellipses tend to align with the mean PV contours in the northwestern part of the subtropical gyre, in the eastern part of the domain they cross the PV contours, resulting in the overall poor correlation between the orientations of the mean PV contours and the direction of spreading ellipses (Fig. 18) (mean PV contours from several depth levels and isopycnal surfaces yielded to a similar result.) The RMS of the difference between their orientations is between $25^{\circ}$ and $60^{\circ}$. Poor correlation was also found between the orientations of the ellipses and contours of $f / H$ with water depth $H$ and Coriolis parameter $f$. This suggests importance of factors other than the local mean PV structure in explaining the preferred direction of the eddy-induced particle dispersion. The nonlocal nature of our estimates, however, may complicate this issue. Additionally, the correlation of the particle dispersion with the oscillating PV contours can potentially be different from the correlation with the mean PV contours. We leave this kind of analysis for the future.

How can the diffusive model be used to represent the eddy-induced spreading in non-eddy-resolving simulations? Since neutrally-buoyant particles represent a passive tracer, the Lagrangian particle dispersion is often used to guide diffusion-based parameterization of the eddy-induced tracer fluxes. The eddy-induced particle spreading is strongly affected by the mean currents and these effects need to be accounted for in parameterization of eddy transports. Because of this, we think that the EFFT diffusivity estimates, which characterize the eddyinduced particle spreading in the presence of the mean currents, are better suited for this purpose than the EO estimates (see also the appendix). However, the utility of the EFFT diffusivities for parameterizing eddy effects in non-eddy-resolving models needs to be tested further 


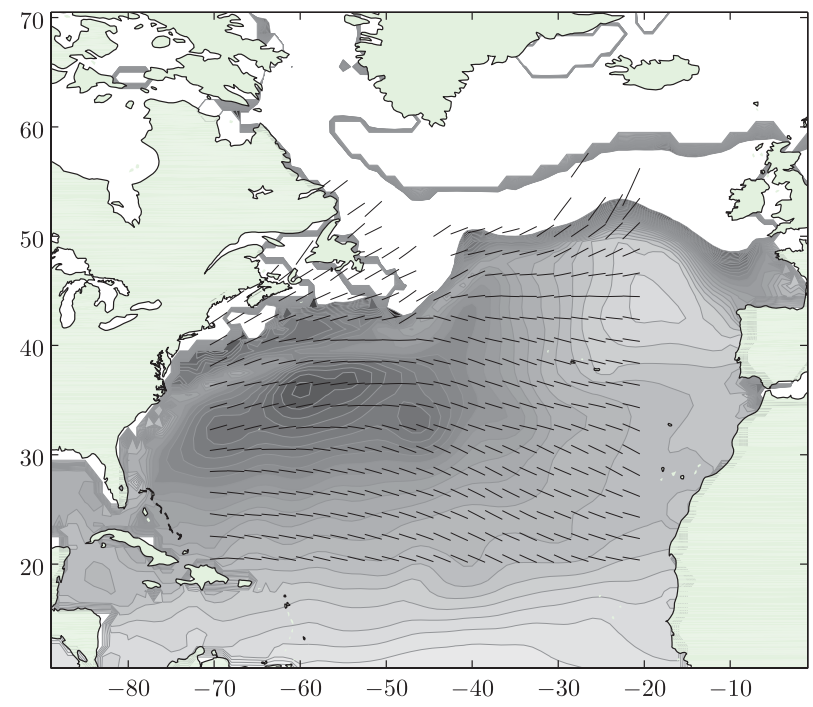

FIG. 18. Tilts of the eddy-fillowing-full-trajectory spreading ellipses (black lines) superimposed on contour levels of PV on a 27.1 potential density surface. PV values have been computed using $f \partial \rho / \partial z$, where density $\rho$ is a locally referenced potential density, derived from monthly Levitus values, and extrapolated onto a $\sigma_{\theta}=27.1$ potential density surface (referenced to the surface). Regions where this isopycnal does not exist (high latitudes and land) are left blank. Other methods, including computing PV from potential density and/or analyzing PV on z-levels, produced similar results.

to see whether EFFT diffusivities can indeed replace eddy advection. Some of the caveats associated with the EFFT estimates are due to spatial nonlocality, and the not exactly diffusive character of the spreading. To make more local estimates, diffusivities should be estimated while the particles are still close to each other and the spreading is induced by the local eddy field. However, on longer times that are required for reaching the diffusive regime, the particles spread too far apart and the estimate becomes substantially nonlocal. We also find that even at longer times ( $>180$ days), in several parts of the domain, the particle spreading substantially deviates from the diffusion; however, it remains unclear whether these deviations are due to locally nondiffusive properties. In particular, in agreement with Berloff et al. (2002), the region of the Gulf Stream extension corresponds to subdiffusive (slower than diffusive) spreading, that we argue may be explained by a local maximum of eddy intensity in this region. The finite bin size $\left(1^{\circ} \times 1^{\circ}\right)$ and the nonlocal nature of the spreading estimates smooth away small-scale structure of transport properties, such as sharp inhomogeneities and possible transport barriers. In this respect, using smaller bins might be preferential, but it will remedy neither the spatial nonlocality nor the nondiffusive character of the spreading. Other techniques for estimating local spreading rates, perhaps based on tracer distribution, may prove to be more accurate in this regard.

Our estimated ranges of diffusivities are $\left(0.06 \leq K_{\tau} \leq\right.$ 12) $\times 10^{4} \mathrm{~m}^{2} \mathrm{~s}^{-1}$ and $\left(0.02 \leq K_{n} \leq 0.5\right) \times 10^{4} \mathrm{~m}^{2} \mathrm{~s}^{-1}$ with $\left\langle K_{\tau}\right\rangle_{\text {mean }}=0.8 \times 10^{4} \mathrm{~m}^{2} \mathrm{~s}^{-1}$ and $\left\langle K_{n}\right\rangle_{\text {mean }}=0.2 \times$ $10^{4} \mathrm{~m}^{2} \mathrm{~s}^{-1}$ with subscripts $\tau$ and $n$ denoting the directions along and across the direction of fastest spreading. These values are consistent with our analysis of GDP drifters and are in general agreement with past diffusivity estimates in Zhurbas and Oh (2004) $[(0.015 \leq K \leq$ 2.6) $\left.\times 10^{4} \mathrm{~m}^{2} \mathrm{~s}^{-1}\right]$, Lumpkin et al. (2002) $[(0.013 \leq K \leq$ 2.1) $\times 10^{4} \mathrm{~m}^{2} \mathrm{~s}^{-1}$ ], and Kamenkovich et al. (2009a) $\left[(0.04 \leq K \leq 1) \times 10^{4} \mathrm{~m}^{2} \mathrm{~s}^{-1}\right]$.

Submesoscale and ageostrophic motions near the surface can affect particle spreading. However, our analysis suggests that the Ekman velocity is less important in dispersing particles than the intermediate-scale geostrophic eddies, since the standard and Ekman-inclusive runs result in similar outcomes. Given the limited data coverage of the GDP dataset, it is also premature to interpret the differences between our altimetric and GDP estimates as manifestation of the importance of smallscale and ageostrophic motions. These differences can be caused by, for example, insufficient spatial and temporal coverage of GDP drifters in some part of the domain, biases in the mean advection estimated from the drifters, different time intervals over which diffusivities were estimated, as well as by the mismatch in the temporal content of the GDP and altimetric datasets. In particular, if the biases in the mean advection were large, substantial part of the large-scale mean advection would be present in the "eddies", thus, resulting in faster (superdiffusive) dispersion. Estimating diffusivities over shorter time intervals may also lead to faster dispersion. The superdiffusive spreading of GDP drifters in the south-eastern part of the subtropical gyre suggests, therefore, that the GDP dataset may have a significant bias in this region.

Understanding the importance of eddies in distribution of oceanic tracers is a challenging problem. Our data analysis study demonstrates complexity of the eddyinduced particle dispersion, which is characterized by spatial inhomogeneity and anisotropy, as well as by nondiffusive behavior. It remains unclear how important these effects are for a particular tracer distribution; and more studies targeted at a specific tracer and/or regime of the flow are needed. The demonstrated complexity of the eddy effects shows importance of resolving mesoscale motions in numerical simulations and observational datasets.

Acknowledgments. The authors thank Kevin Speer and two anonymous reviewers for constructive criticism that helped to improve this manuscript. IR was supported by Grant NSF-OCE-0725796. IK would like to 

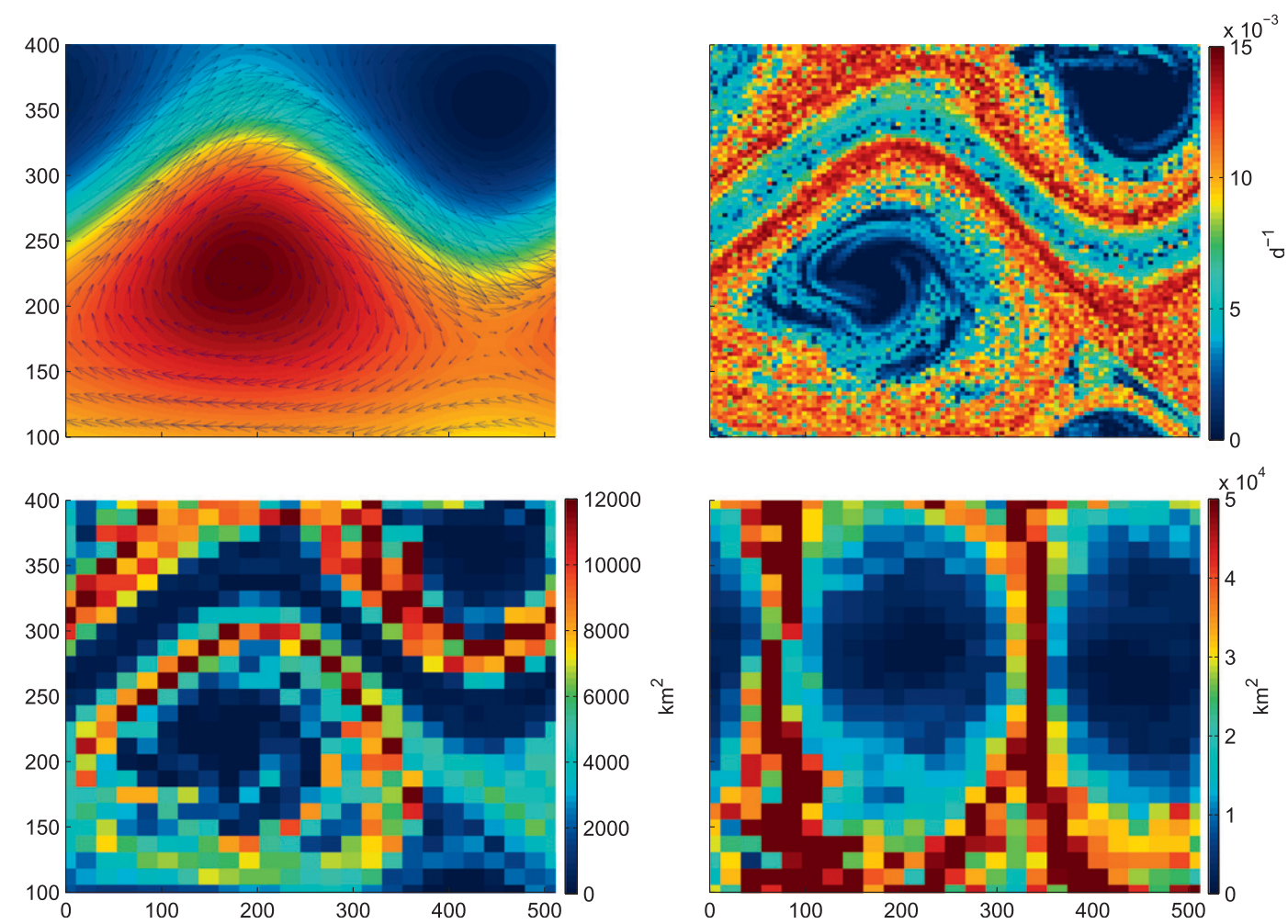

FIG. A1. For a numerically generated flow that has a form of a strong nonsteady meandering jet: (top left) a snapshot of a streamfunction and the velocity field at $t=0$ days; (top right) a snapshot, at $t=0$ days, of the forwardtime Lyapunov exponents computed with integration time $T_{\text {int }}=500$ days; (bottom left) EFFT meridional singleparticle dispersion, $D_{y}^{\mathrm{EFFT}}$, after 500 days for an ensemble of particles released at $t=0$; and (bottom right) EO meridional single-particle dispersion, $D_{y}^{\mathrm{EFFT}}$, after 500 days for an ensemble of particles released at $t=0$.

acknowledge support by the National Science foundation Grant OCE-0842834.

\section{APPENDIX}

\section{Application of the EFFT Method to a Numerically Generated Meandering Jet Flow}

In this appendix we test the ability of the EFFT method to account for the influence of the mean flow on the eddy-induced dispersion of particles. In particular, we are investigating whether the EFFT technique can capture the suppression of the cross-stream material transport in flows with strong mean jets (Smith 2005; Ferrari and Nikurashin 2010; Rypina et al. 2007a,b, 2011). The flow field that we make use of in this appendix has a form of a strong eastward meandering jet that evolves in time. This single-jet solution was obtained with the twolayer quasigeostrophic model described in Berloff et al. (2011) for eastward-background flow case, with zero bottom friction, eddy viscosity $1 \mathrm{~m}^{2} \mathrm{~s}^{-1}$, domain size $520 \times 520 \mathrm{~km}$, on $512^{2}$ grid (i.e., grid scale is about $1 \mathrm{~km}$ ), layer depths 1 and $3 \mathrm{~km}, \mathrm{Rd}=25 \mathrm{~km}$, beta-lane with $\beta=2 \times 10^{-11} \mathrm{~m}^{-1} \mathrm{~s}^{-1}$, starting from very small random noise, with double-periodic boundary conditions, flat bottom. After initial spinup (about 50 years) solution reached statistical equilibrium. For this solution, a snapshot of the streamfunction together with the corresponding velocity field is shown in Fig. A1 (top left). For this flow, the finite-time Lyapunov exponents or FTLEs (Fig. A1, top right) show a meandering ribbon (blue) of smaller FTLE values near the center of the jet, which indicates inhibited stirring in this region. In contrast, the large FTLE (red) areas directly above and below the jet correspond to enhanced stirring and indicate regions that are in the state of chaotic advection [see, for example, Rypina et al. (2007a,b) for more details]. The jet separates these two chaotic zones from each other and acts to suppress the fluid exchange between them. In agreement with this picture, the EFFT meridional single-particle dispersion $D_{y}^{\mathrm{EFFT}}$ [Fig. A1 (bottom left)] is also small near the center of the jet, suggesting that, in this example, the EFFT method captures the suppression of the eddyinduced meridional transport across the jet. In contrast, EO method (bottom right) misses this effect and yields to a very different spatial pattern. 


\section{REFERENCES}

Andersson, M., K. A. Orvik, J. H. LaCasce, I. Koszalka, and C. Mauritzen, 2011: Variability of the Norwegian Atlantic current and associated eddy field from surface drifters. J. Geophys. Res., 116, C08032, doi:10.1029/2011JC007078.

Armi, L., and D. B. Haidvogel, 1982: Effects of variable and anisotropic diffusivities in a steady-state diffusion model. J. Phys. Oceanogr., 12, 785-794.

Bauer, S., M. S. Swenson, and A. Griffa, 2002: Eddy mean flow decomposition and eddy diffusivity estimates in the tropical Pacific Ocean: 2. Results. J. Geophys. Res., 107, 3154, doi:10.1029/2000JC000613.

Berloff, P., J. C. McWilliams, and A. Bracco, 2002: Material transport in oceanic gyres. Part I: Phenomenology. J. Phys. Oceanogr., 32, 764-796.

— I. Kamenkovich, and J. Pedlosky, 2009: A model of multiple zonal jets in the oceans: Dynamical and kinematical analysis. J. Phys. Oceanogr., 39, 2711-2734.

_- S. Karabasov, T. Farrar, and I. Kamenkovich, 2011: On latency of multiple zonal jets in the oceans. J. Fluid Mech., 686, 534-567.

Booth, J., and I. Kamenkovich, 2008: Isolating the role of transient mesoscale eddies in mixing of a passive tracer in an eddy resolving model. J. Geophys. Res., 113, C05021, doi:10.1029/ 2007JC004510.

Chelton, D., M. G. Schlax, and R. M. Samelson, 2011: Global observations of nonlinear mesoscale eddies. Prog. Oceanogr., 91, 167-216, doi:10.1016/j.pocean.2011.01.002.

Davis, R. E., 1991: Observing the general circulation with floats. Deep-Sea Res., 38, 531-571.

Ferrari, R., and M. Nikurashin, 2010: Suppression of eddy diffusivity across jets in the southern ocean. J. Phys. Oceanogr., 40, 1501-1519.

Garraffo, Z., A. J. Mariano, A. Griffa, C. Veneziani, and E. Chassignet, 2001: Lagrangian data in a high resolution numerical simulation of the North Atlantic. I: Comparison with in-situ drifter data. J. Mar. Syst., 29, 157-176.

Gille, S., and R. Davis, 1999: The influence of mesoscale eddies on coarsely resolved density: An examination of subgrid-scale parameterization. J. Phys. Oceanogr., 29, 1109-1123.

Griesel, A., S. T. Gille, J. Sprintall, J. L. McClean, J. H. LaCasce, and M. E. Maltrud, 2010: Isopycnal diffusivities in the Antarctic circumpolar current inferred from Lagrangian floats in an eddying model.J. Geophys. Res., 115, C06006, doi:10.1029/2009JC005821.

Huang, H.-P., A. Kaplan, E. Curchitser, and N. Maximenko, 2007: The degree of anisotropy for mid-ocean currents from satellite observations and an eddy-permitting model simulation. J. Geophys. Res., 112, C09005, doi:10.1029/2007JC004105.

Kamenkovich, I., P. Berloff, and J. Pedlosky, 2009a: Anisotropic material transport by eddies and eddy-driven currents in a model of the North Atlantic. J. Phys. Oceanogr., 39, 3162-3175.

$\longrightarrow, \ldots$, and $-2009 \mathrm{~b}$ : Role of eddy forcing in the dynamics of multiple zonal jets in a model of the North Atlantic. J. Phys. Oceanogr., 39, 1361-1379.

Keating, S. R., K. S. Smith, and P. R. Kramer, 2011: Diagnosing lateral mixing in the upper ocean with virtual tracers: Spatial and temporal resolution dependence. J. Phys. Oceanogr., 41, 1512-1534.

Klocker, A., R. Ferrari, J. H. LaCasce, and S. T. Merrifield, 2012a: Reconciling Lagrangian and eulerian estimates of eddy diffusivities: Tracer and particle-based estimates. J. Mar. Res., in press.
- - — and - 2012b: Estimating suppression of eddy mixing by mean flows. J. Phys. Oceanogr., 42, 1566-1576.

Krauss, W., and C. Boning, 1987: Lagrangian properties of eddy fields in the northern North Atlantic as deduced from satellitetracked buoys. J. Mar. Res., 45, 259-291.

LaCasce, J. H., 2000: Floats and f/h. J. Mar. Res., 58, 61-85.

_ 2008: Statistics from Lagrangian observations. Prog. Oceanogr., 77, 1-29.

_ barotropic flows. J. Mar. Res., 57, 245-274.

, and A. Bower, 2000: Relative dispersion in the subsurface North Atlantic. J. Mar. Res., 58, 863-894.

Lumpkin, R., A.-M. Treguier, and K. Speer, 2002: Lagrangian eddy scales in the northern Atlantic Ocean. J. Phys. Oceanogr., 32, $2425-2440$.

Marshall, J., E. Shuckburgh, H. Jones, and C. Hill, 2006: Stability and implications of surface eddy diffusivity in the southern ocean derived from tracer transport. J. Phys. Oceanogr., 36, 1806-1821.

Maximenko, N. A., B. Bang, and H. Sasaki, 2005: Observational evidence of alternating zonal jets in the world ocean. Geophys. Res. Lett., 32, L12607, doi:10.1029/2005GL022728.

McClean, J. L., P.-M. Poulain, J. W. Pelton, and M. E. Maltrud, 2002: Eulerian and Lagrangian statistics from surface drifters and a high-resolution pop simulation in the North Atlantic. J. Phys. Oceanogr., 32, 2472-2491.

Morrow, R., R. Coleman, J. Church, and D. Chelton, 1994: Surface eddy momentum flux and velocity variances in the Southern Ocean from Geosat altimetry. J.Phys. Oceanogr., 24, 20502071.

Nakamura, M., and Y. Chao, 2000: On the eddy isopycnal thickness diffusivity of the Gent-Mcwilliams subgrid mixing parameterization. J. Climate, 13, 502-510.

Nakamura, N., 1996: Two-dimensional mixing, edge formation, and permeability diagnosed in the area coordinate. J. Atmos. Sci., 53, 1524-1537.

Naveira Garabato, A. C., R. Ferrari, and K. L. Polzin, 2011: Eddy stirring in the southern ocean. J. Geophys. Res., 116, C09019, doi:10.1029/2010JC006818.

O’Dwyer, J., R. G. Williams, J. H. LaCasce, and K. G. Speer, 2000: Does the potential vorticity distribution constrain the spreading of floats in the North Atlantic? J. Phys. Oceanogr., 30, 721-732.

Okubo, A., 1971: Oceanic diffusion diagram. Deep-Sea Res., 18, 789-802.

Owens, W. B., 1984: A synoptic and statistical description of the gulf stream and subtropical gyre using sofar floats. J. Phys. Oceanogr., 14, 104-113.

Preisendorfer, R. W., 1988: Principal Component Analysis in Meteorology and Oceanography. Elsevier, $444 \mathrm{pp}$.

Ralph, E. A., and P. P. Niiler, 1999: Wind-driven currents in the tropical Pacific. J. Phys. Oceanogr., 29, 2121-2129.

Roberts, M., and D. Marshall, 2000: On the validity of downgradient eddy closures in ocean models. J. Geophys. Res., 105, $28613-28627$.

Rypina, I. I., M. G. Brown, F. J. Beron-Vera, H. Kocak, M. J. Olascoaga, and I. A. Udovydchenkov, 2007a: On the Lagrangian dynamics of atmospheric zonal jets and the permeability of the Stratospheric Polar Vortex. J. Atmos. Sci., 64, $3593-3610$

and $2007 b$ : Robust transport barriers resulting from strong Kolmogorov-Arnold-Moser stability. Phys. Rev. Lett., 98, 104102, 10.1103/PhysRevLett. 98.104102. 
— L. J. Pratt, and M. S. Lozier, 2011: Near-surface transport pathways in the North Atlantic Ocean. J. Phys. Oceanogr., 41, 911-925.

Sallee, J.-B., K. Speer, R. Morrow, and R. Lumpkin, 2008: An estimate of Lagrangian eddy statistics and diffusion in the mixed layer of the southern ocean. J. Mar. Res., 66, 441-463.

Scott, R. B., B. K. Arbic, C. L. Holland, B. Qiu, and A. Sen, 2008: Zonal versus meridional velocity variance in satellite observations and realistic and idealized ocean circulation models. Ocean Modell., 23, 102-112.

Smith, K. S., 2005: Tracer transport along and across coherent jets in two-dimensional turbulent flow. J. Fluid Mech., 544, $133-142$.

Vallis, G. K., 2006: Atmospheric and Oceanic Fluid Dynamics. Cambridge University Press, 745 pp.
Veneziani, M., A. Griffa, A. M. Reynolds, Z. D. Garraffo, and E. P. Chassignet, 2005: Parameterizations of Lagrangian spin statistics and particle dispersion in the presence of coherent vortices. J. Mar. Res., 63, 1057-1083.

Von Kameke, A., F. Huhn, G. Fernandez-Garcia, A. P. Mumuzuri, and V. Perez-Munuzuri, 2011: Double cascade turbulence and richardson dispersion in a horizontal fluid flow induced by faraday waves. Phys. Rev. Lett., 107, 074502, doi:10.1103/ PhysRevLett.107.074502.

Young, R., and S. Jones, 1991: Shear dispersion. Phys. Fluids A, 3, 1087-1101.

Zhurbas, V., and I. Oh, 2004: Drifter-derived maps of lateral diffusivity in the Pacific and Atlantic Oceans in relation to surface circulation patterns. J. Geophys. Res., 109, C05015, doi:10.1029/2003JC002241. 